\title{
Low-frequency, low-magnitude vibrations (LFLM) enhances chondrogenic differentiation potential of human adipose derived mesenchymal stromal stem cells (hASCs)
}

Krzysztof Marycz, Daniel Lewandowski, Krzysztof A Tomaszewski, Brandon Michael Henry, Edward B Golec, Monika Marędziak

The aim of this study was to evaluate if low-frequency, low-magnitude vibrations (LFLM) could enhance chondrogenic differentiation potential of human adipose derived mesenchymal stem cells (hASCs) with simultaneous inhibition of their adipogenic properties for biomedical purposes. We developed a prototype device that induces lowmagnitude $(0.3 \mathrm{~g})$ low-frequency vibrations with the following frequencies: 25,35 and 45 $\mathrm{Hz}$. Afterwards, we used human adipose derived mesenchymal stem cell (hASCS), to investigate their cellular response to the mechanical signals. We have also evaluated hASCs morphological and proliferative activity changes in response to each frequency. Induction of chondrogenesis in hASCs, under the influence of a $35 \mathrm{~Hz}$ signal leads to most effective and stable cartilaginous tissue formation through highest secretion of Bone Morphogenetic Protein 2 (BMP-2), and Collagen type II, with low concentration of Collagen type I. These results correlated well with appropriate gene expression level.

Simultaneously, we observed significant up-regulation of $\alpha 3, \alpha 4, \beta 1$ and $\beta 3$ integrins in chondroblast progenitor cells treated with $35 \mathrm{~Hz}$ vibrations, as well as Sox-9. Interestingly, we noticed that application of $35 \mathrm{~Hz}$ frequencies significantly inhibited adipogenesis of hASCs. The obtained results suggest that application of LFLM vibrations together with stem cell therapy might be a promising tool in cartilage regeneration. 
1 Low-frequency, low-magnitude vibrations (LFLM) enhances chondrogenic differentiation

2 potential of human adipose derived mesenchymal stromal stem cells (hASCs).

3

4 Krzysztof Marycz 1, 2, Daniel Lewandowski ${ }^{3}$, Krzysztof A Tomaszewski ${ }^{4,5}$, Brandon Michael

5 Henry $^{4}$, Edward B. Golec ${ }^{5,6}$, Monika Marędziak ${ }^{7}$

7 1. Faculty of Biology, University of Environmental and Life Sciences, Kozuchowska 5b St, 50-

8631 Wroclaw, Poland

9 2. Wroclaw Research Centre EIT +, Stablowicka 147 St, 54-066 Wroclaw, Poland

10 3. Institute of Materials Science and Applied Mechanics, University of Technology, 11 Smoluchowskiego 25 St, 50-370 Wroclaw, Poland

12 4. Department of Anatomy, Jagiellonian University Medical College, Kopernika 12 St, 31-034

13 Krakow, Poland

14 5. Department of Orthopaedics and Trauma Surgery, 5th Military Clinical Hospital and 15 Polyclinic, Krakow, Poland

16 6. Orthopaedic Rehabilitation Department, Chair of Clinical Rehabilitation, Bronislaw Czech 17 Academy of Physical Education, Krakow, Poland

18 7. Faculty of Veterinary Medicine, Department of Animal Physiology and Biostructure 19 University of Environmental and Life Sciences, Norwida 31 St, 50-375 Wroclaw, Poland 20

21 Corresponding author: Krzysztof Marycz, Electron Microscopy Laboratory, Kożuchowska 5b, 22 51-631 Wroclaw, Poland, krzysztofmarycz@interia.pl 


\section{Abstract}

25 The aim of this study was to evaluate if low-frequency, low-magnitude vibrations (LFLM) could

26 enhance chondrogenic differentiation potential of human adipose derived mesenchymal stem

27 cells (hASCs) with simultaneous inhibition of their adipogenic properties for biomedical

28 purposes. We developed a prototype device that induces low-magnitude $(0.3 \mathrm{~g})$ low-frequency

29 vibrations with the following frequencies: 25, 35 and $45 \mathrm{~Hz}$. Afterwards, we used human adipose

30 derived mesenchymal stem cell (hASCS), to investigate their cellular response to the mechanical

31 signals. We have also evaluated hASCs morphological and proliferative activity changes in

32 response to each frequency. Induction of chondrogenesis in hASCs, under the influence of a 35

$33 \mathrm{~Hz}$ signal leads to most effective and stable cartilaginous tissue formation through highest

34 secretion of Bone Morphogenetic Protein 2 (BMP-2), and Collagen type II, with low

35 concentration of Collagen type I. These results correlated well with appropriate gene expression

36 level. Simultaneously, we observed significant up-regulation of $\alpha 3, \alpha 4, \beta 1$ and $\beta 3$ integrins in

37 chondroblast progenitor cells treated with $35 \mathrm{~Hz}$ vibrations, as well as Sox-9. Interestingly, we

38 noticed that application of $35 \mathrm{~Hz}$ frequencies significantly inhibited adipogenesis of hASCs. The

39 obtained results suggest that application of LFLM vibrations together with stem cell therapy

40 might be a promising tool in cartilage regeneration. 


\section{Introduction}

43 Articular cartilage injuries are a growing problem in both human and veterinary medicine. Injury

44 to cartilage manifests through the typical signs of inflammation, and can be caused by either

45 trauma or diseases such as osteonecrosis, cartilage necrosis, or arthritis. Because cartilage is an

46 avascular tissue with chondrocytes that are characterized by a low mitotic potential, the

47 regenerative potential cartilage is substantially limited [1]. As such, the spontaneous regeneration

48 of injured cartilage is extremely difficult. Until recently, the vast majority of the available

49 treatment methods have focused on eliminating symptoms and improving patient quality-of-life

50 through the use steroidal or non-steroidal anti-inflammatory drug treatment (NSAIDs) [2].

51 However, when used long-term, these medications may lead to chondronecrosis [3].

52 A potential solution to this problem emerges in the form of cell based therapies. Adult

53 mesenchymal stem cells (MSCs) may be a possible source of cells for this type of therapy due to

54 their immunomodulatory action, ability to self-renew, and ability to differentiate into several cell

55 lineages, i.e. chondrocytes, osteoblasts or adipocytes [4, 5]. Currently, bone marrow (BMMSCs)

56 and adipose derived mesenchymal stem cells (ASCs) are the cells most frequently applied in

57 cell-based therapies at the preclinical stage. Of the two cell types mentioned above, ASCs seem a

58 better alternative to BMMSCs, due to their easy accessibility, and thus lower donor-related risks

59 [6]. Moreover, activated ASCs secrete from their surface small, spherical membrane fragments

60 called microvesicles (MVs) [7]. These MVs contain important regenerative molecules, that

61 improve the function of damaged tissues - eg. growth factors, bioactive lipids, proteins.

62 Microvesicles secreted by MSCs, stimulated to differentiate into osteocytes, release into the

63 culture medium compounds rich in Collagen type I and II or Bone Morphogenetic Protein 2 [8, 
64 9]. Several studies have confirmed the beneficial clinical effect of ASCs in the treatment of 65 musculoskeletal disorders, particularly in the field of veterinary orthopedics [10, 11, 12]. In our 66 previous study, we demonstrated the positive effects of ASCs application in equine and canine 67 osteoarthritis treatment [13].

68 However, one major limitation to the clinical application of ASCs is the age-related decrease in 69 the proliferative and chondrogenic differentiation potential of ASCs obtained from older 70 populations [14]. As degenerative joint diseases increase in prevalence with age, it is important to develop methods to overcome this limitation. In order to improve both the proliferative and 72 the differentiation potential of ASCs, various physical stimuli such as static magnetic field [15], 73 electric signals [16] and cyclic strain [17] have been applied. However, this field still remains 74 relatively unexplored in terms of the biological effects of low-magnitude low-frequency 75 vibration (LMLF) - a non-invasive biophysical intervention that leads to cyclic loading of the 76 targeted tissue. LMLF vibrations include values of magnitude under $1.0 \mathrm{~g}$, where $\mathrm{g}=9,81 \mathrm{~m} / \mathrm{s}^{2}$, 77 and a frequency between $20-100 \mathrm{~Hz}$ [18]. Several studies have investigated the role of various magnitudes and frequencies of vibrations, such as high-magnitude low-frequency (HMLF) 79 vibrations [19], high-magnitude high frequency vibrations (HMHF) [20] and low-magnitude high-frequency (LMHF) vibrations [21], in the context of their influence on cellular response 81 [22-26]. Moreover, it has been reported that LMHF enhance the osteogenic differentiation 82 potential of MSCs [20]. Enhancement of osteogenic and/or chondrogenic differentiation 83 potential of MSCs may strongly depend on up-regulation of particular integrins, that are 84 activated by various biomechanical signals [27]. Integrins are heterodimeric glycoproteins that 85 are composed of an $\alpha$ - and a $\beta$-subunit, each of which has an extracellular and a cytoplasmic 86 domain [28]. Several studies have provided evidence that chondrocytes express integrins [29- 
87 34]. In particular, the $\alpha 1 \beta 1$ and $\alpha 5 \beta 1$ integrins have been shown to be the most prominent in 88 adult chondrocytes isolated from normal articular cartilage. However, the other integrins are still

89 poorly investigated, especially in the context of their expression in differentiated precursor cells

90 additionally stimulated by various types of external mechanical or others signals.

91 In an animal model, LMHF signals had a positive influence on both bone formation and density, 92 enhancing bone strength and recovery after bone fracture [35, 36, 37]. Moreover, preliminary 93 studies in children with disabling conditions and post-menopausal women indicate that such

94 signals can be efficacious in reversing and/or preventing bone loss [37]. However, to the best 95 knowledge of the authors, the current literature lacks data concerning the effects of LMLF 96 vibrations on the chondrogenic differentiation potential of human ASCs.

97 The aim of this study was to investigate how harmonic vibration, sinusoidal with constant low98 magnitude $\left(0.3 \mathrm{~g}\right.$, where $\left.\mathrm{g}=9,81 \mathrm{~m} / \mathrm{s}^{2}\right)$ and low-frequency $(25,35,45 \mathrm{~Hz})$ mechanical signals, 99 generated by an actuating device, effects ASC morphology, growth, and adipogenic and 100 chondrogenic differentiation potential. 


\section{Materials and Methods}

\section{Description of the cell vibration generator prototype}

104 The process of inducing vibrations was applied using custom-made vibration platforms, specially

105 constructed device that allowed to induce mechanical motion of a 24-well culture plate.

106 Movement of the plate was characterized by the harmonic sine of a given amplitude and

107 frequency. The direction of plate translations was perpendicular to the main surface on which the 108 cells were cultured.

109 A scheme of the stand is shown in Fig. 1C and photographs in Figure 1A, 1B. The vibration

110 generator, an electromagnetic actuator, is positioned on a stationary base. The principle of its

111 operation is based on coil movement, which is generated by an alternating current flow. The

112 design of the actuator is similar to that of a typical loudspeaker, with the main difference being

113 that the moving parts are not a flexible membrane but a stiff plate.

114 The stiff plate of the actuator moves in a linear manner in relation to the stationary part. Fig. 1C

115 depicts the displacement value as ' $\mathrm{x}$ '. Between the actuator and the culture plate, a spacer has

116 been mounted. The spacer is a rigid element made of polyethylene, placed to create distance

117 from the cell culture actuator. This was done to eliminate the possible influence of the alternating

118 magnetic field generated by the EM-actuator on the cell culture. The height of the spacer was

119 about $10 \mathrm{~cm}$. The strength of the magnetic field at this distance does not differ from the 120 background.

121 The culture plate was attached to the top surface of the spacer in such a way to allow quick

122 mounting. Such a method was dictated by the fact that the vibration stimulation was scheduled

123 only for short periods of time each day. Movement of the culture plate was defined as a course of 124 the sine function with a given value of frequency and amplitude of acceleration. A laser 
125 displacement sensor (KEYENCE LK-G157) was used to measure the translation of the culture

126 plate. The acceleration signal was calculated according to the following formula:

$$
x=A \sin (\omega t) \quad \rightarrow \quad \ddot{x}=-A \omega^{2} \sin (\omega t)
$$

128 where $x$ - displacement, $\ddot{x}$ - acceleration, $A$ - amplitude of displacement, $\omega$ - frequency of vibrations $(\omega=2 \pi f), f$

129 - frequency, $t$ - time, $A \omega^{2}$ - amplitude of acceleration.

\section{Vibration loading protocol of hASCs culture}

131 The hASCs were seeded at a concentration of $3 \times 10^{4}$ on 24 - well plates and $5 \times 10^{4}$ to a $15 \mathrm{ml}$

132 tube. Tubes with 3D model were vibrated on tube rack. For each vibration model (25, 35 and

$13345 \mathrm{~Hz}$ ) separate dishes were used. Plates/racks were placed securely onto the vibration device

134 and oscillated vertically at 25, 35 and $45 \mathrm{~Hz}$. The stimulus was sinusoidal and delivered with a

135 peak acceleration of $0,3 \mathrm{~g}$ for 15 minutes once a day, for 14 consecutive days. Cells in the non-

136 vibration group were placed on the same but stationary plate. After 15 min of vibration, the

137 hASCs (both vibrated and non-vibrated groups) received fresh culture medium.

\section{Isolation of human adipose derived mesenchymal stem cells (hASCs)}

139 This study was approved by the local bioethics committee of Wroclaw Medical University,

140 Poland (number KB-177/2014). Written informed consent was obtained from each patient prior

141 to tissue collection during total hip arthroplasty. This study adhered to the Helsinki Declaration

142 (1964) and its later amendments.

143 Subcutaneous adipose tissue was collected from 4 patients. From each patient we obtained 4

144 donor samples, representing of a total $n=16$. The average age of the patients was $69 \pm 1$ years.

145 Briefly, after collection, the tissue samples were placed in sterile Hank's Balanced Salt Solution

146 (HBSS). The isolation procedure of adipose-derived mesenchymal stem cells was conducted 
147 under aseptic conditions and in accordance with previously described protocol [38, 39]. Samples

148 were washed with HBSS supplemented with a $1 \%$ antibiotic-antimicotic solution

149 (penicillin/streptomycin/amphotericin $\mathrm{b}$ at a concentration of $0.017 \mathrm{~mol} / 1,0.01 \mathrm{~mol} / 1$ and

$1500.0002 \mathrm{~mol} / 1$ respectively; Sigma Aldrich, cat no A5955) and then cut into small pieces using

151 surgical scissors. Next, the samples were placed in a sterile centrifuge tube and digested with

152 type I collagenase ( $1 \mathrm{mg} / \mathrm{ml}$, Sigma Aldrich, cat no C5894). After 30 minutes incubation at $37^{\circ} \mathrm{C}$,

153 the tissue homogenate was centrifuged at $1200 \mathrm{~g}$ for 10 minutes. The supernatant was removed

154 and the cell pellet was resuspended in growth media. The cell suspension was then transferred to

155 the cell culture flask.

\section{Immunophenotyping, Fluorescence-activated cell sorting (FACs) analysis, and multipotency}

157 test

158 Cells were plated on 24-well culture plates suspended in $500 \mu 1$ of standard medium at a

159 concentration of $8 \times 10^{3}$ cells per well. The presence of specific antigens for ASCs, i.e. integrin

160 beta-1 (CD29), HCAM (CD44), 5'-nucleotidase (CD73) and endoglin (CD105) and leukocyte

161 common antigen (CD45) was examined after one week of culture by means of primary

162 antibodies (all from Sigma Aldrich). Negative staining of CD45 was used to exclude

163 hematopoietic origin. After fixation, cells were permeabilized with $0,2 \%$ Tween 20 for 15

164 minutes and washed three times with HBSS. The solution of primary antibody and 4\% FBS in

165 PBS was applied to every well and incubated overnight at $4^{\circ} \mathrm{C}$. Next, the cells were washed three

166 times and secondary goat anti-rabbit conjugated with Atto 488 antibody was added to

167 appropriate wells at concentration 1:150. After incubation at room temperature for 1.5 hours, the 168 cells were washed again and photographed under a fluorescence microscope. 
169 For the multipotency test, cells were cultured on chondrogenic and adipogenic media

170 (STEMPRO ${ }^{\circledR}$ Chondrogenesis/Osteogenesis Differentiation Kit and STEMPRO® Adipogenesis

171 Differentiation Kit, Life Technologies) for 14 days. The culture media was changed every

172 second day.

173 After 3 passages, the ASCs were examined for surface protein molecule expression by flow

174 cytometry. Cells were trypinized using a Trypsin-EDTA solution (TrypLE ${ }^{\text {TM }}$, Life

175 Technologies), centrifuged at $400 \mathrm{xg}$ for 3 minutes, and then washed with PBS containing 2\%

176 FBS (fetal bovine serum) (Sigma Aldrich). A total of $5 \times 10^{5}$ cells were labeled for 20 min (on

177 ice and dark) with antibodies pre-conjugated with allophycocyanin (APC), peridinin

178 chlorphyllprotein (PerCP), fluorescein isothiocyanate (FITC) or phycoerythrin (PE). The

179 following CD surface markers were tested: CD34, CD45, CD105, CD90, CD73, CD44, CD29

180 and IgG1 as an isotype control antibody (BD Pharmingen). The samples were analyzed by a

181 Becton Dickinson FACSCalibur flow cytometer. At least ten thousand events were acquired for

182 each CD surface marker. The data was then analyzed using FlowJo X software (Treestar).

\section{Cell culture}

184 Throughout the experiment, hASCs were cultured in aseptic and constant conditions in an

185 incubator at $37^{\circ} \mathrm{C}, 5 \% \mathrm{CO}_{2}$ and $95 \%$ humidity. The cell population was plated in $\mathrm{T}-75$ culture

186 flasks for primary culture and was maintained in Dulbecco's Modified Eagle's Medium

187 (DMEM) with nutrient F-12 Ham (Sigma Aldrich) supplemented with 10\% FBS and 1\% of

188 antibiotic/antimicotic solution at a concentration of $0,017 \mathrm{~mol} / 1,0,01 \mathrm{~mol} / 1$ and $0,0002 \mathrm{~mol} / 1$

189 respectively (Sigma Aldrich, cat no A5955). The culture medium was changed every second day.

190 Human ASCs were passaged using Trypsin-EDTA solution (TrypLE ${ }^{\mathrm{TM}}$, Life Technologies) in 
191 accordance with manufacturer's instruction after reaching about $80-90 \%$ confluence. Cells were

192 passaged three times before use in experiments.

193 Isolated adipose-derived mesenchymal stem cells were divided into 2 groups. The first one was

194 stimulated with chondrogenic medium and the second one with adipogenic medium. The

195 differentiation processes of hASCs were performed using the STEMPRO ${ }^{\circledR}$ Chondrogenesis

196 Differentiation Kit and STEMPRO ${ }^{\circledR}$ Adipogenesis Differentiation Kit (Life Technologies),

197 respectively. Cells were seeded at $3 \times 10^{4}$ on 24 - well plates and $5 \times 10^{4}$ to a $15 \mathrm{ml}$ tube..The

198 stimulation of cells was performed in accordance with the manufacturers' instruction.

199 The chondrogenic culture was maintained in two systems: 2D in 24-well plates for fluorescent,

200 histochemical stainnings, rtPCR analysis and SEM, and 3D for ELISA tests and Focused Ion

201 Beam Scanning Electron Microscope (FIB-SEM, Auriga Compact Crossbeam, Zeiss, Germany).

202 For the 3D system, $2.5 \times 10^{5} \mathrm{hASCs}$ were seeded into $15 \mathrm{ml}$ polypropylene tubes and pelleted.

203 The hASCs were cultured for 14 days as 3D pellets in induction medium STEMPRO ${ }^{\circledR}$

204 Chondrogenesis Differentiation Kit (Life Technologies). The experiment was repeated three

205 times. Both 2D and 3D cultures were incubated two days before starting vibration stimulation.

206 Cell proliferation assay

207 The cell proliferation factor $(\mathrm{PF})$ was evaluated using the Alamar Blue test (TOX-8, Sigma

208 Aldrich) according to the manufacturer's instructions. The culture media was replaced with a

209 medium containing $10 \%$ of resazurin-based dye and incubated for two hours. Afterwards, the

210 supernatants were collected and subjected to absorbance measurement by means of

211 spectrophotometer (SPECTRO StarNano, BMG Labtech) at $600 \mathrm{~nm}$ of wavelength, with a 
212 distraction of $690 \mathrm{~nm}$ of background absorbance. The procedure was performed during the

213 differentiation period at days $2,5,10$, and 14 .

214 A standard curve obtained during the experiment, allowed to estimate the amount of cells. The

215 population doubling time (PDT) was assessed using an on-line calculator (http://www.doubling-

216 time.com/compute.php).

\section{Examination of hASCs morphology}

218 Cell morphology, cellular composition, and culture growth pattern were analyzed using an

219 inverted, fluorescence microscope (AxioObserverA1, Zeiss) and a scanning electron microscope

220 (SEM; EVO LS15, Zeiss).

221 In order to begin observations, after the culture period, cells were fixed with $4 \%$ 222 paraformaldehyde. After 15-minutes permeabilization with $0.2 \%$ Tween, cells were stained using 223 atto-565-labeled phalloidin for 40 minutes to visualize actin filaments. After triple washing, 224 diamidino-2-phenylindole (DAPI) staining was applied for $5 \mathrm{~min}$ to analyze the distribution of 225 cell nuclei. All fluorescence staining was performed at room temperature in the dark. 226 Additionally, Oil Red O staining for adipogenic cultures and Safranin-O staining for 227 chondrogenic cultures were performed to observe fat droplets and chondrocytes, respectively. 228 After fixation, adipogenic samples were treated with $0.1 \%$ Oil Red O solution for 5 min, Plates 229 were rinsed $3 \mathrm{x}$ with water and images of cells on plate were taken. For quantification the 230 percentage of Oil Red O absorption, the dye was extracted by isopropanol and absorbance was 231 determined at $490 \mathrm{~nm}$. Chondrogenic samples were treated with $1 \%$ acetic acid for $10 \mathrm{~min}$ and 232 stained with Safranin-O for 5 minutes. Images were acquired using a Cannon PowerShot digital 233 camera. In order to evaluate the chondrogenic differentiation efficiency, the concentration of 234 proteoglycans was determined, basing on the binding Safranin O to glycosaminoglycans and 235 spectrophotometric measurements at a $470 \mathrm{~nm}$ wavelength and assessment percentage of 236 Safranin O absorption. As 100\% control, we adopted reagent not added to culture. To analyze

237 detailed morphological features of the cells, especially fat droplets, and chondrogenic nodules, 
238 SEM was performed. After fixation, the cells were washed in distilled water and dehydrated in

239 ethanol (concentrations from 50 to $100 \%$, every $5 \mathrm{~min}$ ). Thoroughly dried cells were coated with

240 gold (ScanCoat 6, Oxford), placed in a microscope chamber, and observed using the SE1

241 detector, at $10 \mathrm{kV}$ of filament's tension. To observe morphological features and measure

242 diameters of nodules $(n=6)$ Ion Beam Scanning Electron Microscope (FIB-SEM, Auriga

243 Compact Crossbeam, Zeiss, Germany) obsrvations were performed. Analysis was performed

244 using a focus ion beam detector at magnification of 200X. Diameter of adipocytes ( $\mathrm{n}=6$ ) was

245 measured using Scanning Electron Microscope (SEM; EVO LS15, Zeiss).

\section{Enzyme-linked immunosorbent assays}

247 In order to evaluate the chondrogenic differentiation efficiency on the protein level, the

248 concentration of chondrogenesis-specific markers was investigated. The total concentration of

249 proteins from pellet cultures was determined with enzyme-linked immunosorbent assay

250 (ELISA). For the analysis, cells homogenate were collected on the last day of the experiment.

251 Chondrogenic media was subjected to a BMP-2 ELISA assay (Bone Morphogenic Protein 2

252 Quantikine ELISA Kit, R\&D Systems), and a Col-1 and a Col-2 ELISA assay (Human Collagen

253 alpha-1(I) and (II) chain ELISA Kit, EIAab). All steps of each ELISA tests were performed in

254 accordance with the manufacturer's protocol. Each sample was prepared in duplicate.

255 Spectrofotometric determination was performed using a microplate reader (Spectrostar Nano,

256 BMG Labtech) at a wavelength equal to $450 \mathrm{~nm}$ and with the correction wavelength of $540 \mathrm{~nm}$.

257 The concentration of proteins was presented as a ratio of protein weight and supernatant volume $258(\mathrm{w} / \mathrm{v})$.

259 Quantitative Real-Time Reverse Transcription Polymerase Chain Reaction (qRT-PCR) In

260 order to analyze gene expression, cells after stimulation were rinsed with HBSS and were

261 homogenized with $0.5 \mathrm{ml}$ of TRI Reagent (Sigma Aldrich) directly in the culture well. The total 
262 RNA was isolated using a phenol-chloroform method as previously described [40]. After 263 isolation, total RNA was diluted in DEPC-treated water. The concentration and purity of RNA

264 preparations was determined by absorbance measured at $260 \mathrm{~nm}$ with a nanospectrophotometer

265 (VPA biowave II). Preparation of DNA-free RNA was performed using DNase I RNase-free kit

266 (Thermo Scientific). For each reaction, $100 \mathrm{ng}$ of total RNA was used. Transcription of gDNA-

267 free total RNA to a complementary DNA (cDNA) was reverse transcribed using Moloney

268 Murine Leukemia Virus Reverse Transcriptase (M-MLV RT) and oligo(dT)15 primers

269 (Novazym). RNA purification and cDNA transcription was performed according to

270 manufacturers' instructions. Quantitative Real-Time polymerase chain reaction (qRT-PCR) was

271 performed using $5 \mu \mathrm{l}$ of cDNA in total volume of $20 \mu \mathrm{l}$ by means of SensiFast SYBR \&

272 Fluorescein Kit (Bioline). The reaction was performed at a $500 \mathrm{nM}$ final concentration of 273 primers. The primer sequences used are presented in Table 1. qRT-PCR was performed as

274 described previously [41]. Expression levels of all analyzed genes were normalized for the 275 expression level of glyceraldehyde-3-phosphate dehydrogenase (GAPDH), a housekeeping gene.

\section{Statistical Analysis}

277 All experiments were performed with at least $3(n=3)$ independent experiments (biological 278 replicates, $n \geq 4$ ) measured as quadriplicate or more (technical replicates, $n \geq 4$ ).’).

279 Statistical analysis was performed using GraphPad Prism 5 software. The statistical significance 280 of results was calculated using the one-way analysis of variance (ANOVA) with post-hoc 281 Dunnett's test by means. A P-value of less than 0.05 was considered statistically significant. 


\section{Results}

284 hASCs - FACs analysis, immunophenotyping and multipotency test

285 Flow cytometry analysis revealed that hASCs showed positive labeling for CD29, CD44, CD73, 286 CD105, and CD90 (Fig. 2B). The investigated cells were negatively labeled for two 287 hematopoietic markers: CD34 and CD45 (Fig. 2B). Additionally, immunohistochemical staining 288 confirmed the presence of mesenchymal markers (CD29, CD44, CD73, CD105) and excluded 289 hematopoietic origin (CD45) (Fig. 2A).

290 Moreover, the multipotent character of hASC's was confirmed by abundant osteogenic and 291 adipogenic differentiation. In contrast to cells cultured under control conditions, the presence of 292 osteo nodules, was observed in hASCs cultivated under osteogenic conditions (Fig. 2C).

293 Moreover, mineral calcium deposits visualized by Alizarin Red staining were clearly detected 294 after 3 weeks of osteogenic differentiation. After 2 weeks of culture with an adipogenic inducing 295 media, hASCs developed Oil Red O positive lipid droplets (Fig. 2C), whereas control cultures 296 grown in standard media failed to produce similar results (Fig. 2C).

Proliferation rate (PF) and population doubling time (PDT) of $3 D$ chondroblasts originated from chondro induced hASCs.

299 The proliferative activity, as well as the PDT, were analyzed during the 14 days of hASCs 300 culturing in chondrogenic induction medium exposed to vibration frequencies of 25,35 and $30145 \mathrm{~Hz}$ and in non-vibration control conditions. The obtained data showed that all investigated

302 vibration frequencies influenced the proliferative potential, as well as the PDT, of chondroblasts 303 originated from hASCs (Fig. 3A, B). The percentage of Alamar Blue reduction decreased 304 proportionally with cell count and activity. 
305 Cells cultured under $25 \mathrm{~Hz}$ frequency reached the highest PF after 2 days of incubation and then

306 declined, but on the $10^{\text {th }}$ day of culture reached higher PF and declined again till the $14^{\text {th }}$ day.

307 Moreover, we noticed the longest PDT (277 \pm 25 hours) when cells were stimulated with a $25 \mathrm{~Hz}$

308 frequency. The cells exposed to $35 \mathrm{~Hz}$ vibrations had a higher PF than control cultures at all

309 investigated time points (Fig. 3A). During the analysis, hASCs after chondrogenic differentiation

310 using $25 \mathrm{~Hz}$ vibrations resulted in the lowest proliferation potential, as well as longest PDT, when

311 compared to the other investigated groups.

\section{The morphology of chondroblasts originated from hASCs}

313 After 14 days, formation of chondroblast-specific nodules could be clearly observed (Fig. 4A-P,

314 Fig. 5A-D, Panel B) and showed a strong orange signal after Safranin O staining (Fig. 4I-L).

315 Characteristic chondrocyte-like cells were observed in all of the investigated cultures, however,

316 the cells cultured under the influence of $35 \mathrm{~Hz}$ vibrations more efficiently induced

317 chondrodifferentiation (Fig. 4N). In these samples, nodules had the largest size (Fig. 6A) as well

318 as exhibited the highest absorption of Safranin O (Fig. 6B). Cells cultured under $25 \mathrm{~Hz}$ vibrations

319 absorbed less Safranin O dye and formed nodules with a significantly smaller diameter than

320 samples cultured with $35 \mathrm{~Hz}$ vibrations (Fig. 6A, B). Chondroinduction of cells treated with

$32145 \mathrm{~Hz}$ were comparable to the control group. Although chondro-nodules had similar diameters,

322 the absorption of Safranin $\mathrm{O}$ by cells cultured with $45 \mathrm{~Hz}$ frequencies was significantly higher 323 (Fig. 6A, B). 
324 Quantitative Collagen 1 and 2 (Col-1, Col-2) and Bone Morphogenetic Protein 2 (BMP-2) assay and chondrogenic gene expression analysis (SOX-9, Col-X, Col-II, Runx, Col-I, ACAN)

326 The performed analysis showed an increase in collagen type 2 concentration in comparison to the

327 amount of collagen type I in all groups where vibrations were applied (Fig. 5A, B, Panel A).

328 These results were additionally confirmed by positive chondrogenic differentiation of hASCs.

329 The highest difference on mRNA level between collagen type II and type I was observed in the 330 cultures stimulated with 25 and $35 \mathrm{~Hz}$ vibrations, and in the control culture. The lowest

331 concentration of collagen type II with respect to collagen type I was observed when $45 \mathrm{~Hz}$

332 frequency vibrations were applied (Fig. 5A, B, Panel A). Cells treated with $35 \mathrm{~Hz}$ frequency

333 vibrations tended to secrete significantly higher amounts of BMP-2 in comparison to the other

334 groups (Fig. 5C, Panel A). Exposure to the $25 \mathrm{~Hz}$ stimulation model resulted in secretion of lower

335 a concentration of BMP-2 in comparison to the $35 \mathrm{~Hz}$, however significantly higher when

336 compared to the $45 \mathrm{~Hz}$ frequencies, as well as to the control culture.

337 The quantitative evaluation of the concentration of collagen type I and type II was additionally

338 confirmed by gene expression analysis (Fig. 4C, E, Panel A). The highest activity of collagen

339 type II and its predominance over expression of collagen type I was observed in cells treated

340 with $35 \mathrm{~Hz}$ vibrations. Similarly to the quantitative evaluation, exposure to $25 \mathrm{~Hz}$ vibrations

341 resulted in lower expression of Collagen type II when compared to the $35 \mathrm{~Hz}$ vibrations model.

342 The gene expression of SOX-9 and Col-X, the master transcription factors of chondrogenesis, 343 gradually increased in $35 \mathrm{~Hz}$ treated cells compared to control group (Fig. 4A, B, Panel A). 344 Aggrecan (ACAN) and RUNX2, another chondrogenic markers, significantly increased after 25 $345 \mathrm{~Hz}$ treatment (Fig. 4D, F, Panel A). 
347 In order to sense and translate the applied external mechanical signals, cells express

348 mechanoreceptors on their surface, such as integrins. In our study, we analyzed the expression

349 changes of four alpha $(\alpha 3, \alpha 4, \alpha 5$ and $\alpha \mathrm{V})$ and three beta $(\beta 1, \beta 3$ and $\beta 5)$ integrin subunits (Fig.

350 7). qPCR analysis demonstrated a slight increase of integrin $\alpha 3, \alpha 4, \beta 1$ and $\beta 3$ subunit expression

351 after $25 \mathrm{~Hz}$ stimulation in comparison to control $(0 \mathrm{~Hz})$. We also found that when cells were

352 stimulated with $35 \mathrm{~Hz}$ vibrations, hASCs significantly upregulated integrin $\alpha 3, \alpha 4, \beta 1$ and $\beta 3$

353 subunit. Interestingly, after $35 \mathrm{~Hz}$ stimulation, the highest increase in expression of the $\beta 3$

354 intergrin was observed. With respect to integrin subunits $\alpha 5, \alpha \mathrm{V}$, and $\beta 5$, expression levels were

355 similar between to stimulated groups, however down-regulated as compared to control.

356 Proliferation factor and population doubling time of human adipocytes originated from adipo

357 induced hASCs.

358 The proliferation factor and PDT were determined in the various groups after 14 days of

359 adipogenic induction. The stimulated cultures were characterized by an irregular proliferation

360 rate (Fig. 8A). Stimulation with a $25 \mathrm{~Hz}$ frequency resulted in an increase of PF when compared

361 with the other groups. The highest PF was on day 5, and from that point on a decreasing trend

362 was observed (Fig. 8A). The $25 \mathrm{~Hz}$ frequency group also has the shortest PDT in comparison to

363 other experimental groups (Fig. 8B).

364 In the $35 \mathrm{~Hz}$ and $45 \mathrm{~Hz}$ treatment groups, proliferation remained decreased at days 2, 5 and 10 of

365 culture in comparison to the control and $25 \mathrm{~Hz}$ group, although this difference was not as

366 pronounced as that on day 14. These results were also reflected in the PDT calculations - the 35

$367 \mathrm{~Hz}$ and $45 \mathrm{~Hz}$ cultures had longer time to achieving PDT (Fig. 8B). 


\section{Morphology of ASCs after adipogenic differentiation}

369 Microscopic analysis of adipocytes that originated from hASCs revealed that the $25 \mathrm{~Hz}$

370 vibrations mostly enhanced the adipogenic differentiation in comparison to the other groups (Fig.

371 9I, Panel B). The findings from gene expression analysis showed noticeable increase

372 of PPAR- $\gamma$ and adiponectin (ADIQ) (Fig. 9A, B, Panel A) in 25 and $35 \mathrm{~Hz}$ stimulated groups

373 compared to control. However, qRT-PCR findings also showed similar gene expression of leptin

374 (LEP) (Fig. 9C, Panel A). The adipocytes derived from $25 \mathrm{~Hz}$ stimulated group were highly 375 abundant with large lipid vacuoles, positively stained by Oil Red O staining, and absorbed the 376 highest percentage of dye (Fig. 10A). The smallest adipocyte diameter was observed in the

377 control group (ranges between 60 and $92 \mathrm{um}$ ), while adipocytes treated with $35 \mathrm{~Hz}$ were 378 characterized by the highest average size (ranges between 90 and 119 um) (Fig. 10B).

379

380 


\section{Discussion}

382 Cartilage defects, especially in cases of osteoarthritis, have a serious impact on the patient's 383 quality-of-life and functionality. The increasing prevalence of degenerative joint diseases is

384 explained by the increasing life expectancy of the general population [42]. Tissue-engineering

385 approaches including the application of externally applied signals such as stimulation with

386 electric currents [43, 44], laser [45], or ultrasound vibration [46, 47], are promising tools for

387 overcoming this problem. However, these methods also have several shortcomings, such as

388 emission of high temperature or generation of rarefactional pressure, which may lead to mild

389 heating, coagulative necrosis, tissue vaporization or inducing pulsation of pre-existing gas bodies

$390[48]$.

391 Even more promising is the combination of MSCs therapy with innovative devices that are able

392 to induce particular external signals, which enhance the regeneration of injured tissues. Since

393 stem cells viability, proliferation status, and differentiation potential are widely connected with

394 their regenerative potential $[49,50]$, searching for external stimulating factors that may enhance

395 the mentioned MSCs features, before clinical application seems to be a crucial factor, when

396 MSCs for cartilage regeneration purposes are considered. Furthermore, effective external

397 stimulation may help overcome the age-related decrease in chondrogenic differentiation potential

398 of hASCs that currently poses a limitation to their clinical application in cell-based therapies

399 [14]. Therefore, in the current study, we hypothesized that LMLF may enhance the chondrogenic

400 differentiation potential of hASCs and simultaneously alter the differentiation toward fat tissue.

401 We found that when the PF of hASCs cultured in chondrogenic conditioned medium is

402 considered, both the $25 \mathrm{~Hz}$ and the $35 \mathrm{~Hz}$ vibration frequencies reduce the PF of hASCs.

403 Additionally, the PDT reached the highest level in cells treated with $25 \mathrm{~Hz}$, which strongly 
404 correlates with the obtained PF factor. As recently reported, the PDT of MSCs directly correlates

405 with their replicative senescence, which is linked to the decrease in the size of cell aggregation

406 [51]. In each experimental group, the PDT was higher in comparison with other studies [52].

407 This may be due to the fact that cells were rapidly differentiated into osteoblasts or adipocytes,

408 and that their proliferation activity was significantly reduced. Sepúlveda et al. [53] reported that

409 cell senescence abrogates the therapeutic potential of human mesenchymal stem cells in a lethal

410 endotoxemia model. Therefore, searching for methods that might improve the PDT level seems

411 to be crucial in the context of clinical application of MSCs. Interestingly, although hASCs

412 treated with $35 \mathrm{~Hz}$ had low PF and long PDT, they also developed the largest chondro nodules.

413 Furthermore, the highest concentration of absorbed Safranin O staining was observed in nodules

414 originating from cells treated with $35 \mathrm{~Hz}$ vibration. This likely demonstrates that $35 \mathrm{~Hz}$

415 vibrations induce the highest synthesis of glycosaminoglycans (GAG), and thus indicates that the

416 chondrogenic process is highly efficient.

417 The GAGs have an unquestionable influence on biomechanical properties of cartilage. They 418 serve as an important component of extracellular matrix (ECM), which directly affects the 419 integrity of cartilage. Our data demonstrates that the $35 \mathrm{~Hz}$ vibration model could be applied to 420 stimulate chondrocytes, which originated from hASCs to produce ECM of high biomechanical 421 properties manifested by rich in collagen type II and proteoglycans. The most extensive 422 morphometrical properties, i.e. length and height of single nodules, were observed in 2D culture 423 cells that were treated with the $35 \mathrm{~Hz}$ vibrations protocol. Slightly weaker chondro nodule 424 development, as well as cytoskeleton formation, was observed in the $2 \mathrm{D}$ culture treated with $42525 \mathrm{~Hz}$ vibrations in comparison to the others, as well as to the control group (Figure 4). 
426 In order to determine which of the investigated frequencies has a more significant impact on the

427 process of chondrogenesis, we applied quantitative ELISA and qPCR in order to evaluate the 428 concentration of BMP-2 and the relationship between collagen type I and II in the culture 429 medium. We found in $3 \mathrm{D}$ culture treated with $35 \mathrm{~Hz}$ the highest concentrations of BMP-2 430 (Figure 5), and at the same time -collagen type II, in the $2 \mathrm{D}$ culture treated with $35 \mathrm{~Hz}$ (Figure 431 4). However, $25 \mathrm{~Hz}$ vibrations caused an increase in synthesis, albeit slightly smaller amounts of 432 investigated proteins. Our obtained results stand in good agreement with the findings of van 433 Cashion et al. [54], who reported that low frequency vibrations can induce secretion of BMP-2 in 434 human umbilical cord derived MSCs. It is worth noting that both the $25 \mathrm{~Hz}$ and $35 \mathrm{~Hz}$ vibration 435 stimulated chondrocytes originating from hASCs had elevated synthesis of type II rather than 436 type I collagen. It is well known that elasticity of articular cartilage is dependent on the proper 437 relationship between synthesis and secretion of collagen types I and II. Simultaneously, we 438 observed, that $35 \mathrm{~Hz}$ vibration stimuli in $2 \mathrm{D}$ culture caused up-regulation of sex-determining 439 region $\mathrm{Y}$ protein $(\mathrm{SRY})$-box 9 (SOX9) that is the primary regulator of chondrogenic 440 differentiation on an in vitro as well as an in vivo level. The observed up-regulation of Sox-9, 441 strongly corresponded with elevated expression of collagen type II, which is to be expected as 442 Sox-9 directly regulates expression of collagen gene (COLL II) in chondrogenic progenitor cells, 443 as well as chondrocytes $[55,56]$. Moreover, SOX9 determines functions of RUNX2 and exerts a 444 dominant function over RUNX2 in mesenchymal precursors [57]. In this study, we confirmed 445 this effect; gene expression of SOX9 and RUNX2 are overlapped and enhanced in cells treated 446 with vibrations.

447 The positive effect of LFLM on the functional chondrogenic differentiation process might be 448 explained by up-regulation of the integrin family. Here, we found that both frequencies i.e. 25 
449 and $35 \mathrm{~Hz}$, affect up-regulation of integrin $\alpha 3, \alpha 4, \beta 1$ and $\beta 3$ subunits on the mRNA level, 450 although statistical significance was observed only in $35 \mathrm{~Hz}$ stimulated cells. Furthermore, $\beta 1$ 451 integrin has been shown to play a crucial role in attachment and survival of MSCs during the 452 chondrogenesis process. Furthermore, we observed that LFLM, in general, significantly down 453 regulates integrin $\alpha 5 \beta 5$ expression. Moreover, we observed down-regulation of $\alpha 5$ integrins of 454 MSCs stimulated with all tested frequencies. Thus our results stand in good agreement with 455 Martino and colleagues (2008), that showed involvement of $\alpha 5$ integrins more in osteogenic 456 rather than chondrogenic differentiation process [58].

457 Finally, the effect of vibration loading with particular frequencies on the adipogenic 458 differentiation potential of hASCs was investigated. We found that among all tested frequencies, $45935 \mathrm{~Hz}$ significantly inhibited adipogenesis in hASCs. The obtained results stand in good 460 agreement with the findings by $\mathrm{Oh}$ at al. [59] who reported that 20 and $30 \mathrm{~Hz}$ sub-sonic 461 vibrations inhibited the proliferation of 3T3-L1 preadipocytes, Additionally, we observed the 462 smallest absorption of Oil red O staining when cells were treated with $35 \mathrm{~Hz}$ vibration, However 463 we observed increased number of lipid droplets in $25 \mathrm{~Hz}$ vibration group, but the observed 464 changes were only on a morphological level. In adipogenic gene expression level of we did not 465 observed significant changes between $25 \mathrm{~Hz}$ and other investigated groups. 


\section{Conclusions}

469 In conclusion, the vibration-loading device designed for the purpose of this study successfully 470 generated controlled vibrational forces to hASCs cultured on 24-well, as well as 3D pelleted 471 cells model. Our results indicate that LFLM vibrations differently act on both chondrogenic, as 472 well as adipogenic potential of hASCs. The most important finding of this study suggests that 35 $473 \mathrm{~Hz}$ frequency vibrations enhance chondrogenic potential of hASCs with simultaneous inhibition 474 of hASCs differentiation toward adipocytes. Finally, we conclude that mechanical signals, 475 especially $35 \mathrm{~Hz}$ frequency vibrations might be potentially used in construction of therapeutic 476 devices which may prove useful in the field of articular degenerative diseases treatment.

478 Acknowledgments

479 We thank Marta Jeleń for assistance during in vitro work and cell vibration generator prototype 480 preparation. 


\section{References}

483 1. Chung C, and Burdick JA (2008). Engineering cartilage tissue. Advanced drug delivery 484 reviews, 60(2), 243-262.

485 2. Lin J, Zhang W, Jones A and Doherty M (2004). Efficacy of topical non-steroidal anti486 inflammatory drugs in the treatment of osteoarthritis: meta-analysis of randomised controlled $487 \quad$ trials. bmj, 329(7461), 324.

488 3. Brandt KD: Effects of nonsteroidal anti-inflammatory drugs on chondrocyte metabolism in 489 vitro and in vivo. Am J Med. 1987, 83(5A): 29-34.

490 4. Iyer SS, Rojas M: Anti-inflammatory effects of mesenchymal stem cells: novel concept for 491 future therapies. Expert Opin. Biol. Ther. 2008, 8(5): 569-581.

492 5. Zuk PA, Zhu M, Mizuno H, Huang J, Futrell JW, Katz AJ, Benhaim P, Lorenz HP, Hedrick 493 MH. (2001) Multilineage cells from human adipose tissue: implications for cell-based 494 therapies. Tissue Eng. 7, 211-228.

495 6. Baer PC, Geiger H (2012) Adipose-derived mesenchymal stromal/stem cells: tissue 496 localization, characterization, and heterogeneity. Stem cells 2012, doi:10.1155/2012/812693

497 7. Marędziak M, Marycz K, Lewandowski D, Siudzinska A, Śmieszek A. (2015) Static 498 magnetic field enhances synthesis and secretion of membrane-derived microvesicles (MVs) 499 rich in VEGF and BMP-2 in equine adipose-derived stromal cells (EqASCs)-a new approach 500 in veterinary regenerative medicine. In Vitro Cellular \& Developmental Biology. Animal. $501 \quad 51(3): 230-240$.

502 8. Collino F, Deregibus MC, Bruno S, Sterpone L, Aghemo G, Viltono L, Camussi G (2010) 503 Microvesicles derived from adult human bone marrow and tissue specific mesenchymal stem 504 cells shuttle selected pattern of miRNAs. PloS one, 5(7), e11803. 
505 9. Tetta C, Consiglio AL, Bruno S, Tetta E, Gatti E, Dobreva M, Cremonesi F, Camussi G 506 (2012) The role of microvesicles derived from mesenchymal stem cells in tissue 507 regeneration; a dream for tendon repair? Muscles Ligaments Tendons J. 2(3): 212-221.

508 10. Marycz K, Grzesiak J, Wrzeszcz K, Golonka P. (2012) Adipose stem cell combined with 509 plasma-based implant bone tissue differentiation in vitro and in a horse with a phalanx 510 digitalis distalis fracture: a case report. Veterinarni Medicina 57(11): 610-617; 2012.

511 11. Marycz K, Toker NY, Grzesiak J, Wrzeszcz K, Golonka P. (2012) The therapeutic effect of 512 autogenic adipose derived stem cells combined with autogenic platelet rich plasma in tendons 513 disorders hi horses in vitro and in vivo research. Journal of Animal and Veterinary Advances $514 \quad 11(23): 4324-4331 ; 2012$.

515 12. Brittberg M, Lindahl A, Nilsson A, Ohlsson C, Isaksson O, Peterson L. Treatment of deep 516 cartilage defects in the knee with autologous chondrocyte transplantation. N Engl J Med $517 \quad 1994 ; 331: 889-895$.

518 13. Nicpoń J, Marycz K, Grzesiak J, Śmieszek A, Toker ZY (2014). The advantages of autologus 519 adipose derived mesenchymal stem cells (AdMSCs) over the non-steroidal anti-inflammatory 520 drugs (NSAIDs) application for degenerative elbow joint disease treatment in dogs-Twelve 521 cases. Kafkas Univ Vet Fak Derg, 20(3), 345-350.

522 14. Choudhery MS, Badowski M, Muise A, Pierce J, Harris DT. Donor age negatively impacts 523 adipose tissue-derived mesenchymal stem cell expansion and differentiation. J Transl Med. $524 \quad 2014 ; 12: 8$

525 15. Marędziak M, Marycz K, Śmieszek A, Lewandowski D, Nezir Yaşar Toker (2014) The 526 influence of static magnetic fields on canine and equine mesenchymal stem cells derived 527 from adipose tissue. In Vitro Cell.Dev.Biol. - Animal, 50:562-571. 
528 16. Hammerick KE, James AW, Huang Z, Prinz FB, Longaker MT. (2009) Pulsed direct current

529 electric fields enhance osteogenesis in adipose-derived stromal cells. Tissue Engineering Part $530 \quad$ A, 16(3), 917-931.

531 17. Simmons CA, Matlis S, Thornton AJ, Chen S, Wang CY, Mooney DJ. (2003). Cyclic strain 532 enhances matrix mineralization by adult human mesenchymal stem cells via the extracellular signal-regulated kinase (ERK1/2) signaling pathway. Journal of biomechanics, 36(8), 10871096.

18. Lau E, Al-Dujaili S, Guenther A, Liu D, Wang L, You L (2010). Effect of low-magnitude, high-frequency vibration on osteocytes in the regulation of osteoclasts. Bone, 46(6):1508-15.

19. Nikander, R Kannus P, Dastidar P, Hannula M, Harrison L, Cervinka T, Narra NG, Aktour R, Arola T, Eskola H, Soimakallio S, Heinonen A, Hyttinen J, Sievänen H. (2009) Targeted exercises against hip fragility. Osteoporos. Int. 20, 1321-1328.

20. Tirkkonen L, Halonen H, Hyttinen J, Kuokkanen H, Sievänen H, Koivisto AM, Haimi S (2011). The effects of vibration loading on adipose stem cell number, viability and differentiation towards bone-forming cells. Journal of The Royal Society Interface, 8(65), $1736-1747$.

21. Luu YK, Capilla E, Rosen CJ, Gilsanz V, Pessin JE, Judex S, Rubin CT. (2009) Mechanical stimulation of mesenchymal stem cell proliferation and differentiation promotes osteogenesis while preventing dietary-induced obesity. J. Bone Miner. Res. 24, 50-61.

22. Edwards JH, Reilly GC (2015) Vibration stimuli and the differentiation of musculoskeletal progenitor cells: Review of results in vitro and in vivo. World J Stem Cells. 7(3): 568-582. 
552 24. Sen B, Xie Z, Case N, Styner M, Rubin CT, Rubin J. (2011) Mechanical signal influence on

553 mesenchymal stem cell fate is enhanced by incorporation of refractory periods into the 554 loading regimen. J Biomech. 44(4): 593-9.

555 25. Prè D, Ceccarelli G, Visai L, Benedetti L, Imbriani M, Cusella De Angelis MG, Magenes G 556 (2013) High-Frequency Vibration Treatment of Human Bone Marrow Stromal Cells 557 Increases Differentiation toward Bone Tissue. Bone Marrow Res. 2013: 803450.

558 26. Uzer G, Pongkitwitoon S, Ete Chan M, Judex S. (2013). Vibration induced osteogenic 559 commitment of mesenchymal stem cells is enhanced by cytoskeletal remodeling but not fluid 560 shear. J Biomech. 2013;46:2296-2302.

561 27. Popov C, Burggraf M, Kreja L, Ignatius A, Schieker M Docheva D. (2015). Mechanical 562 stimulation of human tendon stem/progenitor cells results in upregulation of matrix proteins, 563

28. Goessler UR, Bugert P, Bieback K, Stern-Straeter J, Bran G, Sadick H, Hörmann K, Riedel integrins and MMPs, and activation of p38 and ERK1/2 kinases F. In vitro analysis of integrin expression in stem cells from bone marrow and cord blood during chondrogenic differentiation. J Cell Mol Med. 2009;13(6):1175-84.

29. Hering TM. Regulation of chondrocyte gene expression. Front Biosci. 1999;4:D743-61.

30. Hynes RO. Integrins: versatility, modulation, and signaling in cell adhesion. Cell. 1992;69(1):11-25.

31. Giancotti FG, Ruoslahti E. Integrin signaling. Science. 1999;285(5430):1028-32.

32. Albelda SM, Buck CA. Integrins and other cell adhesion molecules. FASEB J. 1990;4(11):2868-80.

573 33. Salter DM, Hughes DE, Simpson R, Gardner DL. Integrin expression by human articular 574 chondrocytes. Br J Rheumatol. 1992;31(4):231-4. 
575 34. Lee J BC, W, Qi WN, Scully SP. The involvement of beta1 integrin in the modulation by

576 collagen of chondrocyte-response to transforming growth factor-beta1. J Orthop Res.

$577 \quad 2002 ; 20(1): 66-75$.

578 35. Xie, L., Rubin, C. and Judex, S. (2008). Enhancement of the adolescent murine

579 musculoskeletal system using low-level mechanical vibrations. J. Appl. Physiol. 104, 1056-

$580 \quad 1062$.

581 36. Wehrle E, Liedert A, Heilmann A, Wehner T, Bindl R, Fischer L, Haffner-Luntzer M, Jakob

582 F, Schinke T, Amling M, Ignatius A (2015) The impact of low-magnitude high-frequency

583 vibration on fracture healing is profoundly influenced by the oestrogen status in mice. Dis

$584 \quad$ Model Mech. 8(1):93-104.

585 37. Rubin C, Judex S, Qin YX. (2006) Low-level mechanical signals and their potential as a non586 pharmacological intervention for osteoporosis. Age Ageing 35, 32.

587 38. Grzesiak J, Marycz K, Czogala J, Wrzeszcz K, Nicpon J (2011) Comparison of behavior, 588 morphology and morphometry of equine and canine adipose derived mesenchymal stem cells 589 in culture. Int J Morphol 29: 1012-1017.

590 39. Marycz K, Smieszek A, Grzesiak J, Donesz-Sikorska A, Krzak-Ros J (2013) Application of 591 bone marrow and adipose-derived mesenchymal stem cells for testing the biocompatibility of 592 metal-based biomaterials functionalized with ascorbic acid. Biomed Mater 8: 065004.

593 40. Chomczynski P, Sacchi N (1987) Single-step method for RNA isolation by acid guanidinium 594 thiocyanate-phenol-chloroform extraction. Anal Biochem 162: 156-159.

595 41. Kim HJ, Im GI. The effects of ERK1/2 inhibitor on the chondrogenesis of bone marrow- and 596 adipose tissue-derived multipotent mesenchymal stromal cells. Tissue Eng Part A $597 \quad 2010 ; 16(3): 851 \mathrm{e} 60$. 
598 42. Raeissadat S, Rayegani S, Babaee M, Ghorbani E (2013): The Effect of Platelet-Rich Plasma 599 on Pain, Function, and Quality of Life of Patients with Knee Osteoarthritis, Pain Res Treat. $600 \quad 2013 ; 2013: 165967$.

601 43. Ciombor DM, Aaron RK. (2005) The role of electrical stimulation in bone repair. Foot Ankle 602 Clin; 10:579-593, vii.

603 44. Foley KT, Mroz TE, Arnold PM, Chandler HC Jr, Dixon RA, Girasole GJ, Renkens KL Jr, 604 Riew KD, Sasso RC, Smith RC, Tung H, Wecht DA, Whiting DM. Randomized, 605 prospective, and controlled clinical trial of pulsed electromagnetic field stimulation for 606 cervical fusion. Spine J. 2008;8:436-442.

607 45. Miloro M, Miller JJ, Stoner JA. Low-level laser effect on mandibular distraction 608 osteogenesis. J Oral Maxillofac Surg. 2007;65:168-176.

609 46. El-Mowafi H, Mohsen M. The effect of low-intensity pulsed ultrasound on callus maturation 610 in tibial distraction osteogenesis. Int Orthop. 2005;29:121-124.

611 47. Taylor KF, Rafiee B, Tis JE, Inoue N. (2007) Low-intensity pulsed ultrasound does not 612 enhance distraction callus in a rabbit model. Clin Orthop Relat Res;459:237-245.

613 48. Miller D, Smith N, Bailey M, Czarnota G, Hynynen K, Makin I, and American Institute of 614 Ultrasound in Medicine Bioeffects Committee (2012): Overview of Therapeutic Ultrasound 615 Applications and Safety Considerations, J Ultrasound Med.; 31(4): 623-634.

616 49. Krampera M, Pasini A, Pizzolo G, Cosmi L, Romagnani S, Annunziato F (2006):

617 Regenerative and immunomodulatory potential of mesenchymal stem cells; Current Opinion 618 in Pharmacology, Volume 6, Issue 4, August 2006, Pages 435-441. 
619 50. Mishra A, Tummala P, King A, Lee B, Kraus M, Tse V, Jacobs CR (2009): Buffered 620 Platelet-Rich Plasma Enhances Mesenchymal Stem Cell Proliferation and Chondrogenic 621 Differentiation Tissue Engineering Part C: Methods, Vol. 15, No. 3: 431-435

622 51. Yoon IS, Chung CW, Sung JH, Cho HJ, Kim JS, Shim WS, Shim CK, Chung SJ, Kim DD. 623 Proliferation and chondrogenic differentiation of human adipose-derived mesenchymal stem 624 cells in porous hyaluronic acid scaffold. J Biosci Bioeng. 2011 Oct;112(4):402-8.

625 52. Hass, R., Kasper, C., Bohm, S., \& Jacobs, R. (2011). Different populations and sources of 626 human mesenchymal stem cells (MSC): a comparison of adult and neonatal tissue-derived 627 MSC. Cell Commun Signal, 9(1), 12.

53. Sepúlveda JC, Tomé M, Fernández ME, Delgado M, Campisi J, Bernad A, González MA (2014). Cell senescence abrogates the therapeutic potential of human mesenchymal stem cells in the lethal endotoxemia model. Stem Cells.

631 54. Cashion A, Caballero M, Halevi A, Pappa A, Dennis RG, van Aalst JA (2014): 632 Programmable Mechanobioreactor for Exploration of the Effects of Periodic Vibratory 633 Stimulus on Mesenchymal Stem Cell Differentiation, BioResearch Open Access, Volume 3, $634 \quad$ Number 1.

635 55. Akiyama H. Control of chondrogenesis by the transcription factor Sox9. Mod. Rheumatol. $636 \quad 2008 ; 18,213-219$

637 56. Bell DM, Leung KK, Wheatley SC, Ng LJ, Zhou S, Ling KW, Sham MH, Koopman P, Tam 638 PP, Cheah KS. SOX9 directly regulates the type-II collagen gene. Nat Genet. 639 $1997 ; 16(2): 174-8$. 
640 57. Zhou G, Zheng Q, Engin F, Munivez E, Chen Y, Sebald E, Krakow D, Lee B. (2006)

641 Dominance of SOX9 function over RUNX2 during skeletogenesis. Proc Natl Acad Sci U S

642 A. 103(50):19004-9.

643 58. Martino MM, Mochizuki M, Rothenfluh DA, Rempel SA, Hubbell JA, Barker TH.

644 Controlling integrin specificity and stem cell differentiation in 2D and 3D environments

645 through regulation of fibronectin domain stability. Biomaterials. 2009;30(6):1089-97.

646 59. Oh ES, Seo YK, Yoon HH, Cho H, Yoon MY, Park JK (2011): Effects of sub-sonic vibration

647 on the proliferation and maturation of 3T3-L1 cells. Life Sci, Jan 17;88(3-4):169-77

648

649 

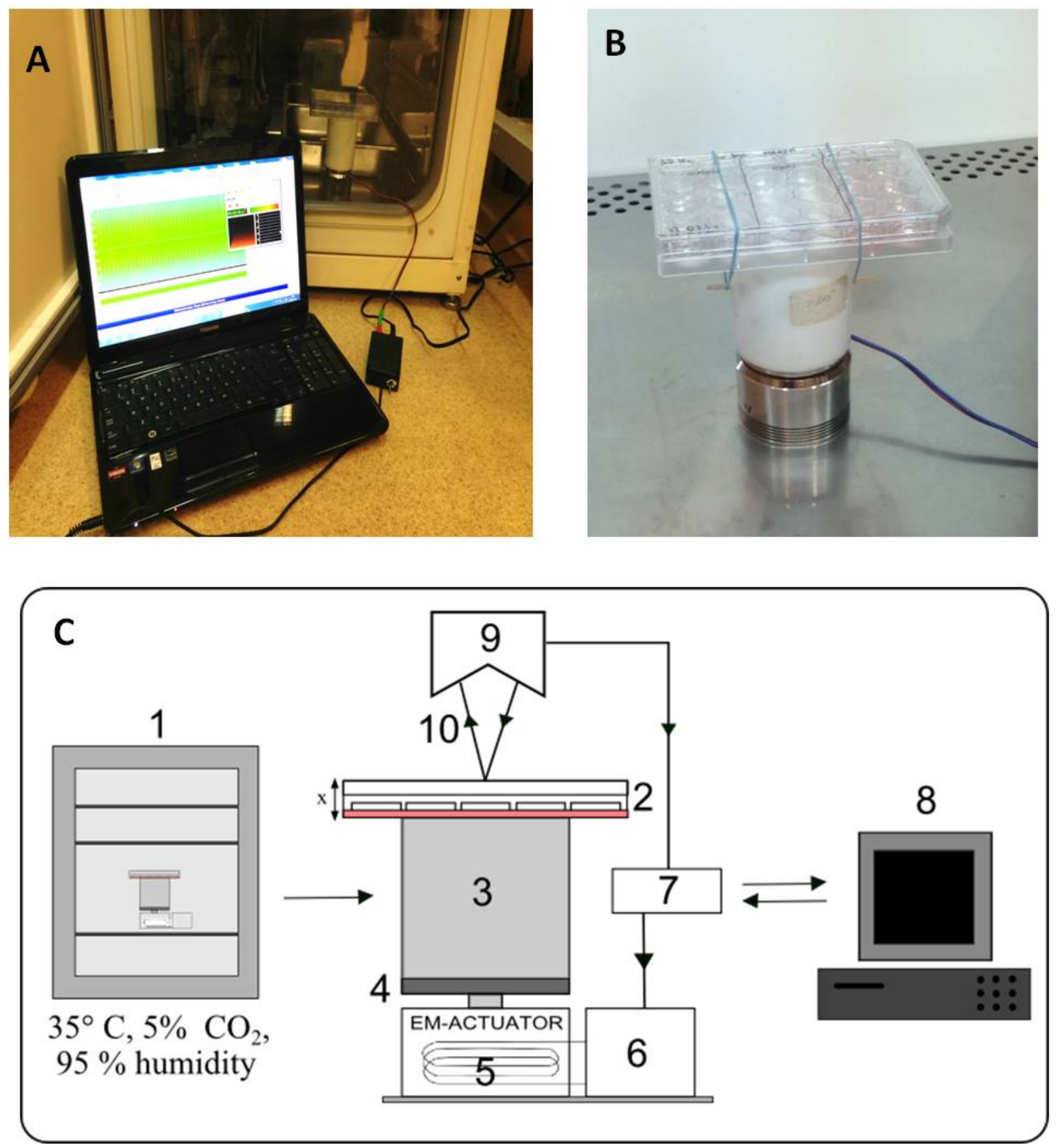

651 Figure 1. Cell vibration generator prototype. Acutator with cell culture plate connected with PC software, put inside

652 a CO2 incubator (A). Cell culture plate attatched to the spacer under electro-magnetic acutator (B). Connection

653 diagram and flow of signals during vibration stimulation. The electromagnetic actuator was supplied directly by the

654 amplifier. The displacement signal waveform was generated in the computer software and sent through a digital-to-

655 analog converter to the amplifier. 1 - incubator, 2 - 24-well culture plate, 3 - spacer, 4 - stiff movable plate, 5 -

656 electro-magnetic actuator (EM-ACTUATOR) with coil inside, 6 - signal amplifier, 7 - measuring card with A/C 
657 and C/A converters, 8 - PC and software, 9 - laser displacement sensor head, 10 - laser beam. $\mathrm{x}$ shows the 658 movement direction of the culture plate $(\mathrm{C})$ 


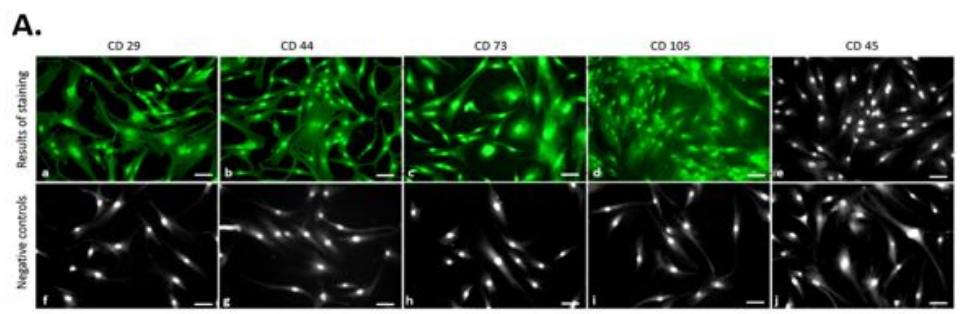

B.
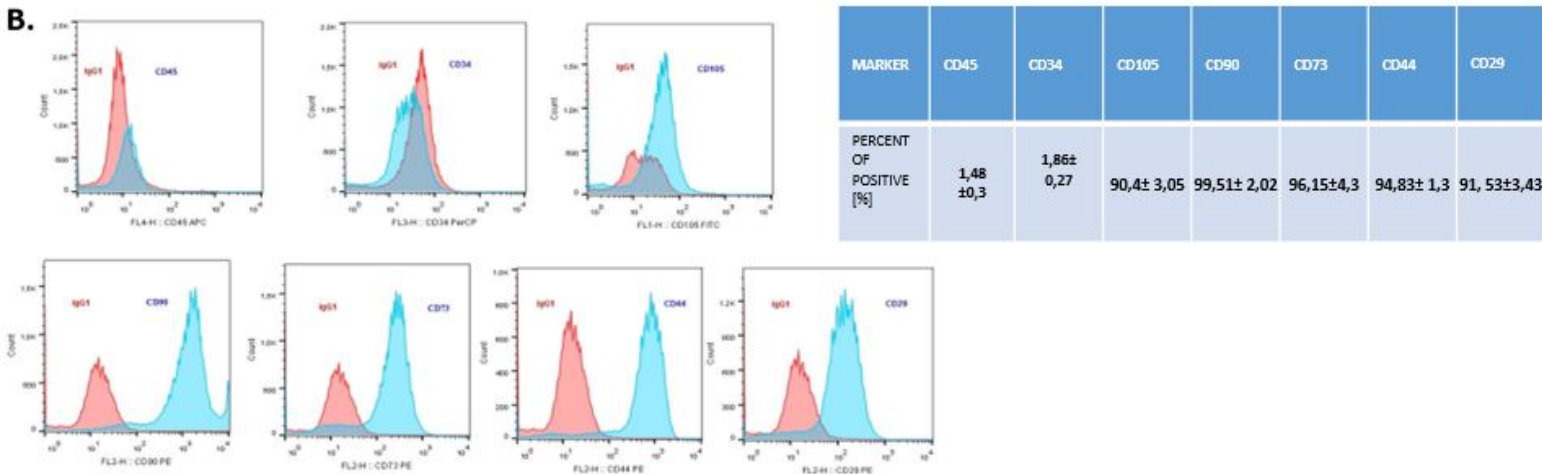

C.

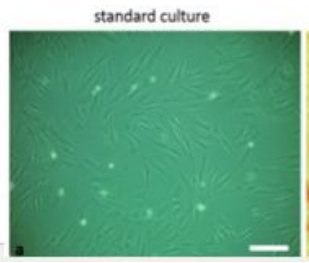

osteogenic stimulation

adipogenic stimulation

659

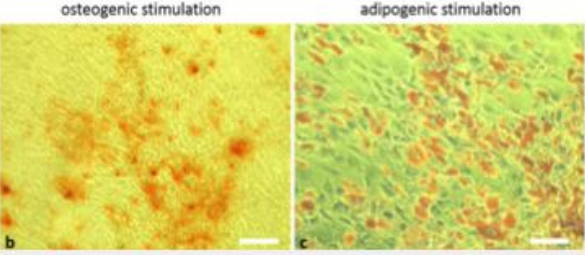

660 Figure 2. The expression of specific cell markers CD29, CD44, CD73 and CD105 and the lack of hematopoetic cell

661 marker CD45 (A). Characterization of hASCs FACS analysis. FACS histograms of passage 3 ASC simultaneously

662 stained for CD45, CD34, CD105, CD90, CD73, CD44, CD29, and IgG1 as negative control. Histograms are

663 representative of 3 independent flow cytometry analyses. Red histograms: IgG1 negative control; blue histograms:

664 antibody specific staining (B). Multipotency assay- standard culture and differentiated cultures after Alizarin Red

665 staining for osteogenic stimulation (mag. 50x, scale bar $=200 \mu \mathrm{m}$ ) and Oil Red O staining for adipogenic

666 stimulation (mag. 100x, scale bar $=400 \mu \mathrm{m})(\mathrm{C})$.

667 


\section{Chondrogenesis proliferation factor and population doubling time}

A

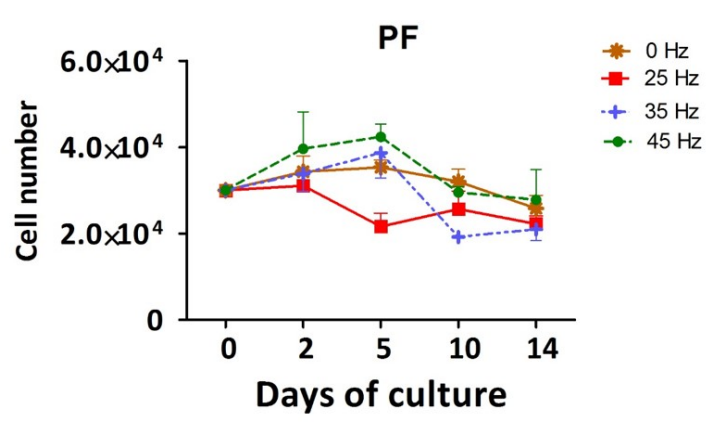

B

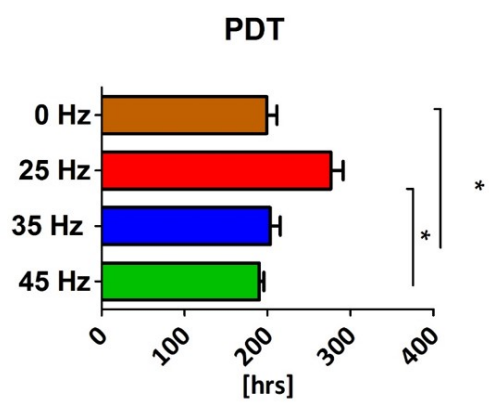

669 Figure 3. Proliferation factor (A) and population doubling time (PDT) (B) of hASCs treated with 0, 25, 35 and 45

$670 \mathrm{~Hz}$ vibration frequencies during chondrogenic stimulation. $*$ p-value $<0.05$.

671 


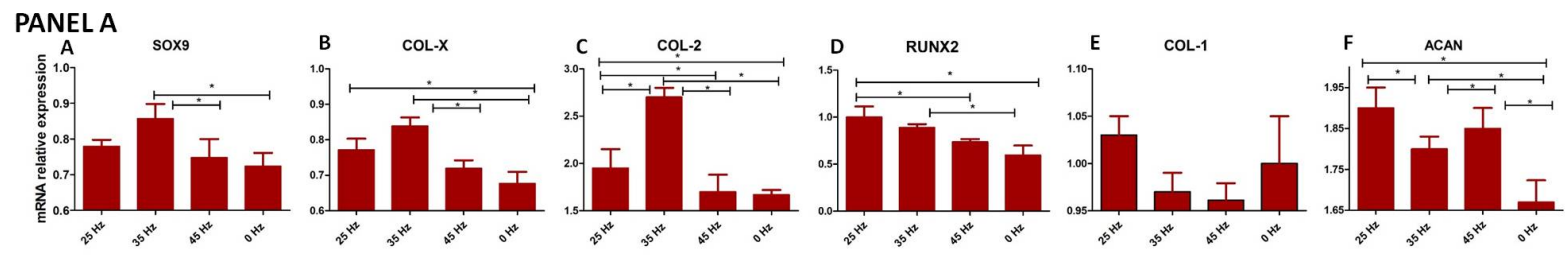

PANEL B

673 Figure 4. Panel A RT-qPCR for chondrogenesis genes: SOX9, COL-X, COL-2, RUNX2, COL-1 and ACAN from

674 hASCs that underwent chondrogenic induction on culture plates with treatment with $0,25,35,45 \mathrm{~Hz}$ vibrations. * p-

675 value $<0.05$ Panel B Cell morphology of chondroblasts originated from hASCs cultured on plates visualized by

676 fluorescence stainings (DAPI A-D and Phalloidin E-H), Safranin staining (I-L). Scale bars A-H $100 \mu \mathrm{m}$ and I-L 200

$677 \mu \mathrm{m}$ and scanning electron microscope photographs (Mag. 2000x).

678 

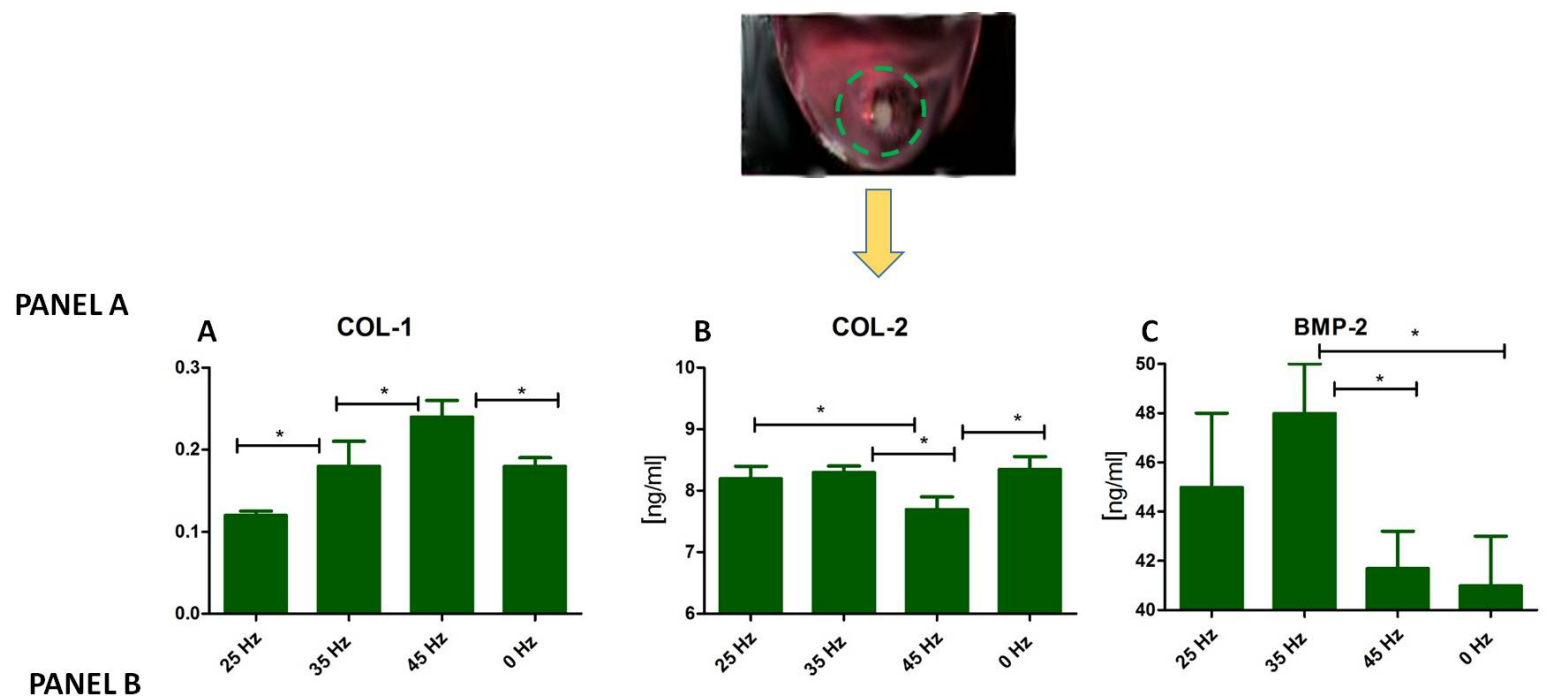

679
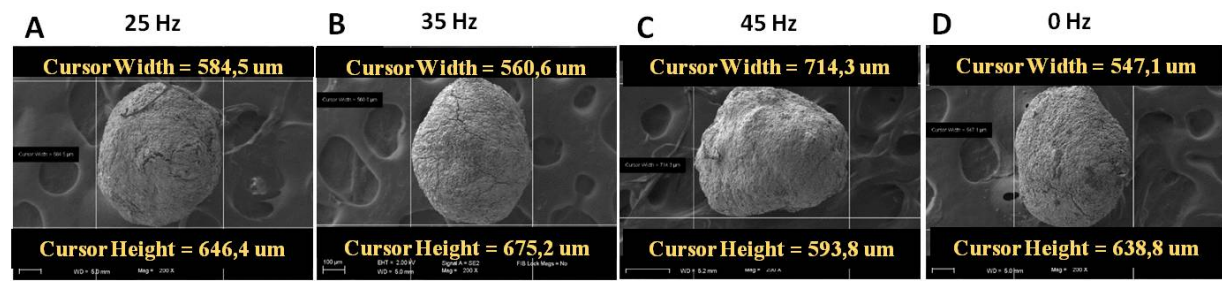

680 Figure 5. Panel A Comparison of Col-1, Col-2 and BMP-2 levels by ELISA hASCs pellets after 14 days of

681 chondro-induction. Panel B Morphological characterization and comparison of chondro-nodules from cells cultured 682 at pellet.

683 
A

Percentage of Safranin staining absorption

684

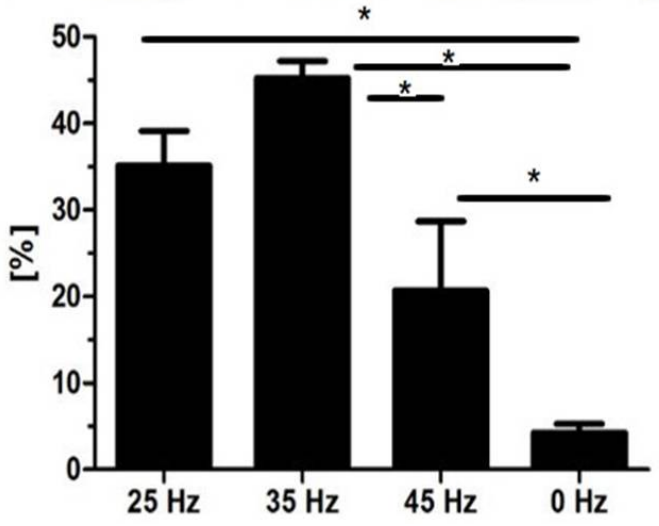

B

The average diameter of nodules

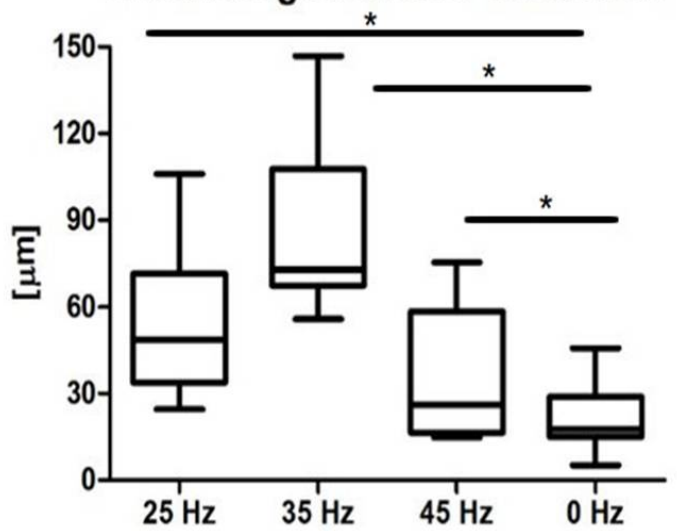

685 Figure 6. Percentage of Safranin staining absorption (A) and the average diameter of chondrogenic nodules (B)

686 from chondroblasts that originated from hASCs. * p-value $<0.05$.

687 

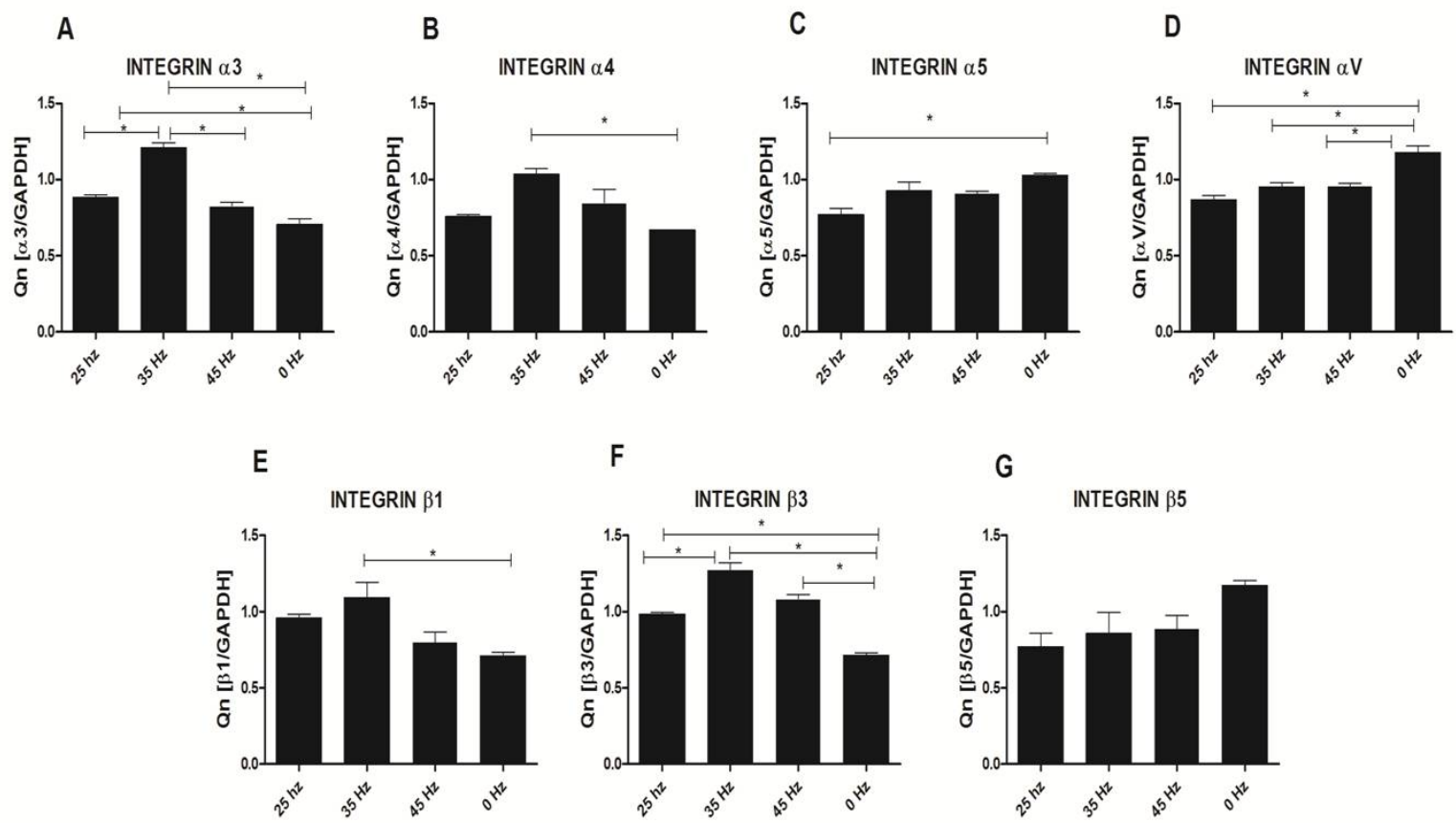

688

689 Figure 7. Integrin expression changes after mechanical stimulation of ASCs. Quantitative PCR analysis for integrin 690 alpha $3,4,5, \mathrm{~V}$ and beta $1,3,5$ subunits. ${ }^{*} \mathrm{p}<0.05$.

691 


\section{Adipogenesis proliferation factor and population doubling time}

A

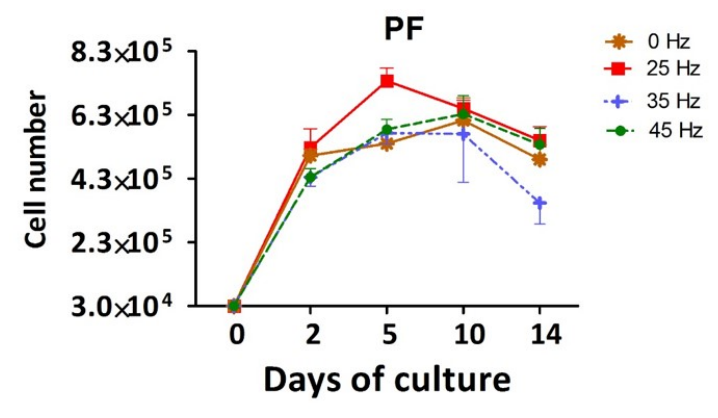

692

B

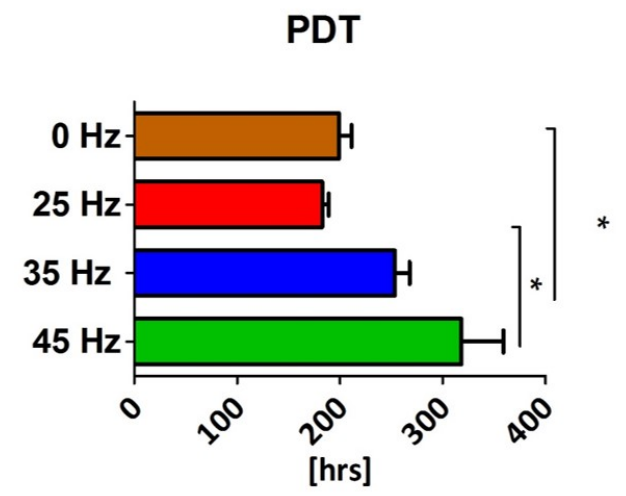

693 Figure 8. Proliferation factor (A) and population doubling time (PDT) (B) of hASCs treated with 0, 25, 35 and 45

$694 \mathrm{~Hz}$ vibration frequencies during adipogenic stimulation. * p-value $<0.05$.

695 
PANEL A

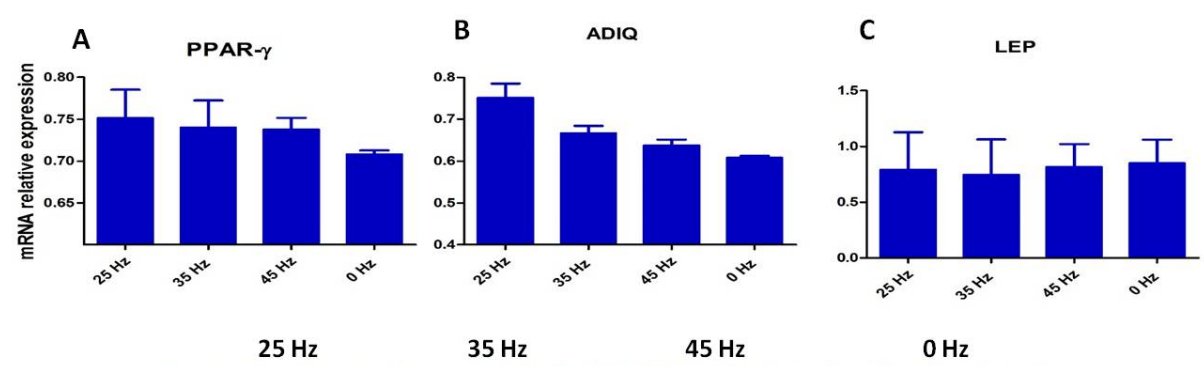

PANEL B

696

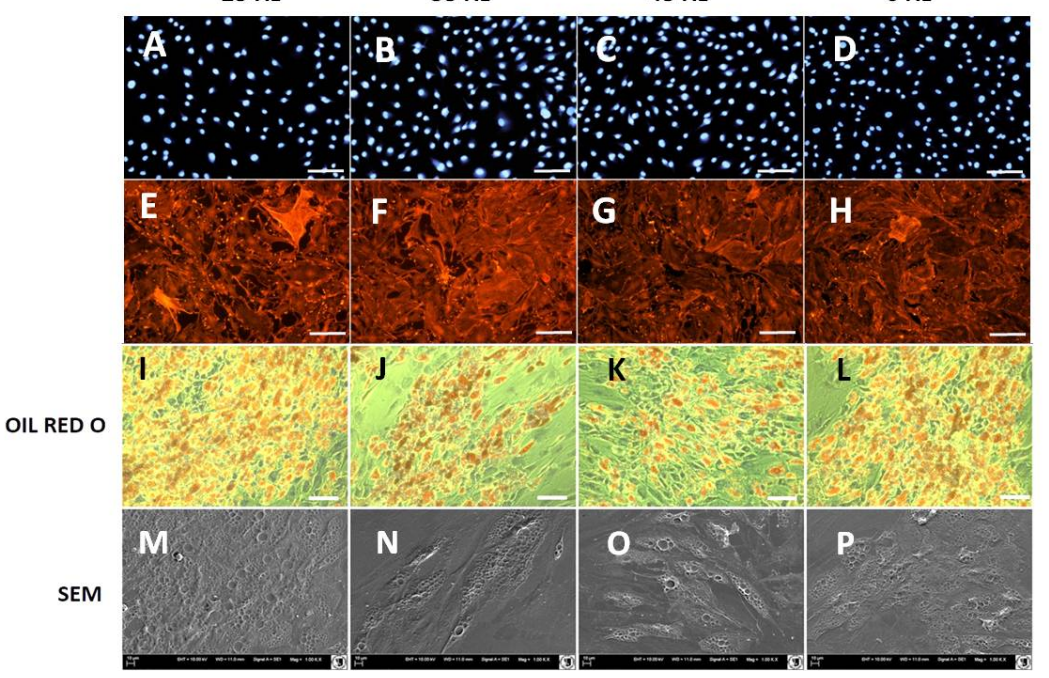

697 Figure 9. Panel A RT-qPCR for adipogenesis genes: PPAR-gamma, ADIQ, LEP from hASCs that underwent

698 adipogenic induction on with treatment with $0,25,35,45 \mathrm{~Hz}$ vibrations. * p-value $<0.05$ Panel B Cell morphology

699 of adipocytes originated from hASCs visualized using fluorescence stainings (Phalloidin, DAPI; Mag. 50x, scale bar

$700200 \mu \mathrm{m})$, Oil Red O Staining $(100 \mathrm{x}$, scale bar $=400 \mu \mathrm{m})$ and scanning electron microscope photographs $(1000 \mathrm{x})$. 
A Percentage of Oil Red 0 staining absorption

702

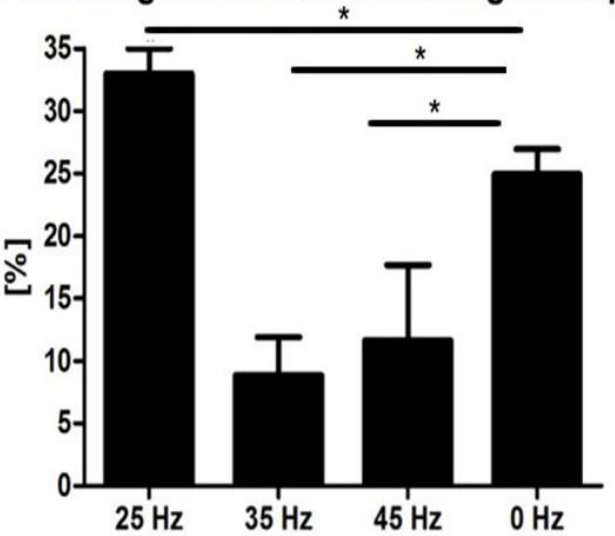

B

The average diameter of adipocytes

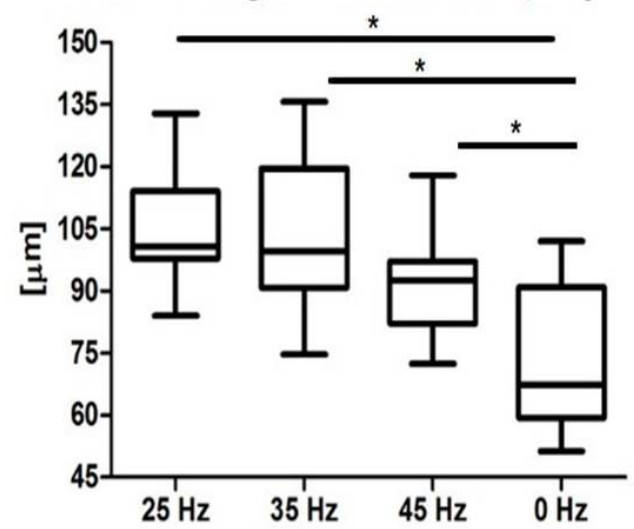

703 Figure 10. Percentage of Oil Red O staining (A) absorption and the average diameter (B) of adipocytes. * p-value $<$ 704 0.05

705

706 


\section{Table 1 (on next page)}

Sequences of $\mathrm{QPCR}$ primers

Sequences of qPCR primers used for the amplification of human mRNA to chondrogenic genes 


\section{Tables:}

\begin{tabular}{|c|c|c|c|}
\hline Gene name & Primer sequentions & $\begin{array}{c}\text { Ann. T, } \\
{ }^{\circ} \mathrm{C}\end{array}$ & $\begin{array}{c}\text { Accession } \\
\text { number }\end{array}$ \\
\hline GAPDH & $\begin{array}{l}\text { Forward 5'- GTCAGTGGTGGACCTGACCT -3' } \\
\text { Reverse 5'- CACCACCCTGTTGCTGTAGC -3' }\end{array}$ & 60 & NM_002046 \\
\hline $\begin{array}{l}\text { Collagen type I } \\
\text { (COL1A1) }\end{array}$ & $\begin{array}{l}\text { Forward 5'- GTGATGCTGGTCCTGTTGGT -3' } \\
\text { Reverse 5'- CACCATCGTGAGCCTTCTCT -3' }\end{array}$ & 60 & NM_000088.3 \\
\hline $\begin{array}{c}\text { Collagen type II } \\
\text { (COL2A1) }\end{array}$ & $\begin{array}{l}\text { Forward 5'- GACAATCTGGCTCCCAAC -3' } \\
\text { Reverse 5'- ACAGTCTTGCCCCACTTAC -3', }\end{array}$ & 60 & NM_001844.4 \\
\hline $\begin{array}{l}\text { Aggrecan } \\
\text { (ACAN) }\end{array}$ & $\begin{array}{l}\text { Forward 5'- GCCTACGAAGCAGGCTATGA -3' } \\
\text { Reverse 5'- GCACGCCATAGGTCCTGA -3' }\end{array}$ & 60 & NM_013227.3 \\
\hline SOX-9 & $\begin{array}{l}\text { Forward 5'-AGCGAACGCACATCAAGAC-3' } \\
\text { Reverse 5'- GCTGTAGTGTGGGAGGTTGAA-3' }\end{array}$ & 65 & NM_000346 \\
\hline RUNX-2 & $\begin{array}{l}\text { Forward 5'- GTGATAAATTCAGAAGGGAGG-3' } \\
\text { Reverse 5'- CTTTTGCTAATGCTTCGTGT-3' }\end{array}$ & 65 & NM_001024630 \\
\hline $\begin{array}{l}\text { Collagen type } X \\
(\text { Col-X) }\end{array}$ & $\begin{array}{l}\text { Forward 5'-CAGTCATGCCTGAGGGTTTT-3' } \\
\text { Reverse 5'-GGGTCATAATGCTGTTGCCT-3' }\end{array}$ & 65 & NM_000493 \\
\hline $\begin{array}{c}\text { Adiponectin } \\
\text { (ADIQ) }\end{array}$ & $\begin{array}{l}\text { Forward 5'-AGGGTGAGAAAGGAGATCC-3' } \\
\text { Reverse 5'- GGCATGTTGGGGATAGTAA-3' }\end{array}$ & 60 & XM_011513324. \\
\hline Leptin (LEP) & $\begin{array}{l}\text { Forward 5'- ATGACACCAAAACCCTCATCAA-3' } \\
\text { Reverse 5'- GAAGTCCAAACCGGTGACTTT-3' }\end{array}$ & 60 & $\begin{array}{l}\text { XM_005250340. } \\
3\end{array}$ \\
\hline PPAR-gamma & $\begin{array}{l}\text { Forward 5'- ATGACACCAAAACCCTCATCAA- 3' } \\
\text { Reverse 5'- GAGCGGGTGAAGACTCATGTCTGTC-3' }\end{array}$ & 60 & AB565476.1 \\
\hline Integrin $\alpha 3$ & \begin{tabular}{|l|} 
Forward 5'-ATCTTGAGAGCCACAGTCA-3' \\
Reverse 5'-cTGGGTCCTTCTTTCTAGTTC-3'
\end{tabular} & 52 & (NM_002204) \\
\hline Integrin $\alpha 4$ & \begin{tabular}{|l|} 
Forward 5'-AATGGATGAGACTTCAGCACT-3' \\
Reverse 5'-CTCTTCTGTTTTCTTCTTGTAGG-3'
\end{tabular} & 58 & (NM_000885) \\
\hline Integrin $\alpha 5$ & $\begin{array}{l}\text { Forward 5'-ACTAGGAAATCCATTCACAGTTC-3' } \\
\text { Reverse 5'-GCATAGTTAGTGTTCTTTGTTGG-3' }\end{array}$ & 52 & (NM_002205) \\
\hline
\end{tabular}




\begin{tabular}{|c|c|c|c|}
\hline Integrin $\alpha v$ & $\begin{array}{l}\text { Forward 5'-GGAGCACATTTAGTTGAGGTAT -3' } \\
\text { Reverse 5'-ACTGTTGCTAGGTGGTAAAACT-3' }\end{array}$ & 56 & (NM_002210) \\
\hline Integrin $\beta 3$ & $\begin{array}{l}\text { Forward 5'-CTGCTGTAGACATTTGCTATGA-3' } \\
\text { Reverse 5'-GCCAAGAGGTAGAAGGTAAATA-3' }\end{array}$ & 52 & (NM_000212) \\
\hline Integrin $\beta 5$ & $\begin{array}{l}\text { Forward 5'-CTGTGGACTGATGTTTCCTT-3' } \\
\text { Reverse 5'-GTATGCTGGTTTTACAGACTCC-3' }\end{array}$ & 54 & (NM_002213) \\
\hline Integrin $\beta 5$ & $\begin{array}{l}\text { Forward 5'- GAAGGGTTGCCCTCCAGA -3' Reverse } \\
\text { 5'- GCTTGAGCTTCTCTGCTGTT-3' }\end{array}$ & 60 & NM_002211.3 \\
\hline
\end{tabular}

2 Table 1. Sequences of qPCR primers used for the amplification of human mRNA to chondrogenic genes. 3 
1

Vibration generation prototype
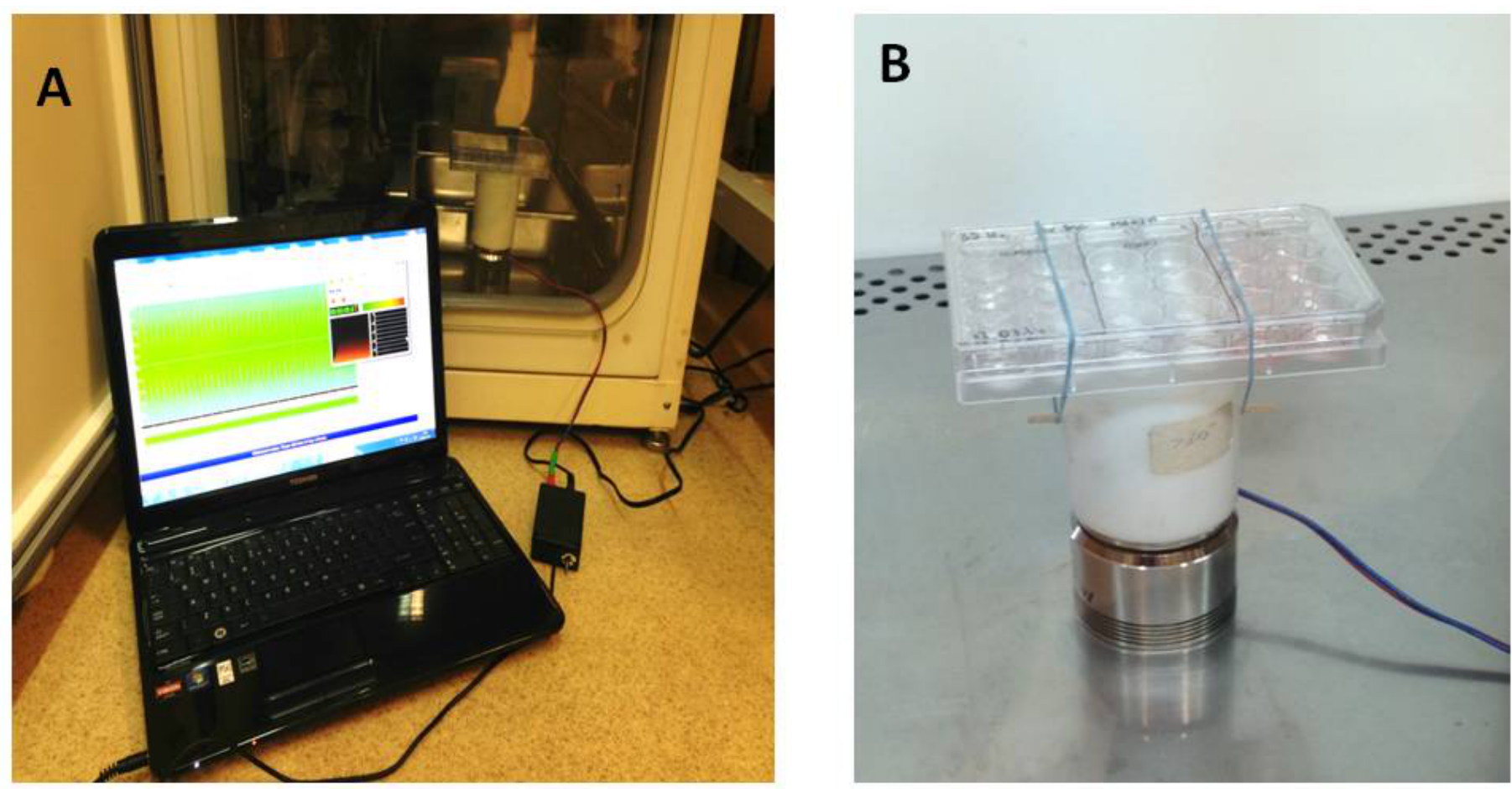

C

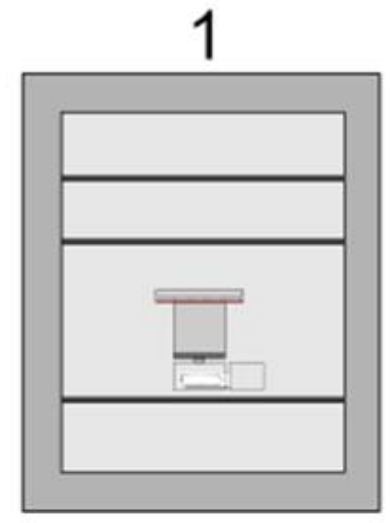

$35^{\circ} \mathrm{C}, 5 \% \mathrm{CO}_{2}$, $95 \%$ humidity

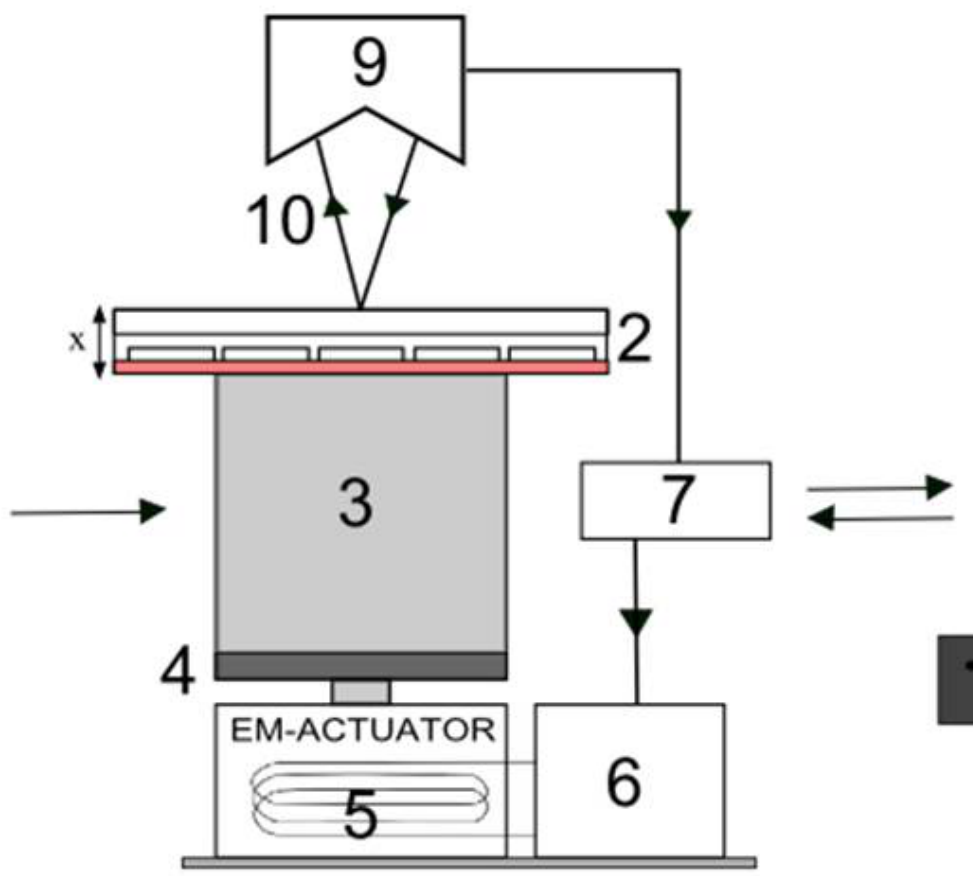

8 


\section{2}

Phenotyping and multipotency test

A.

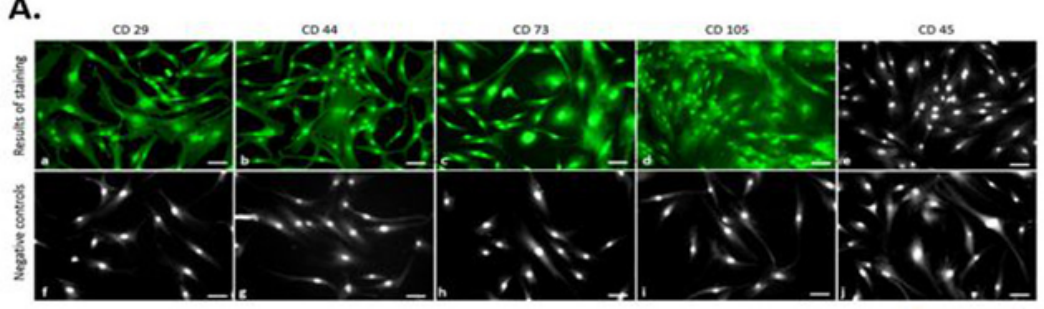

B.
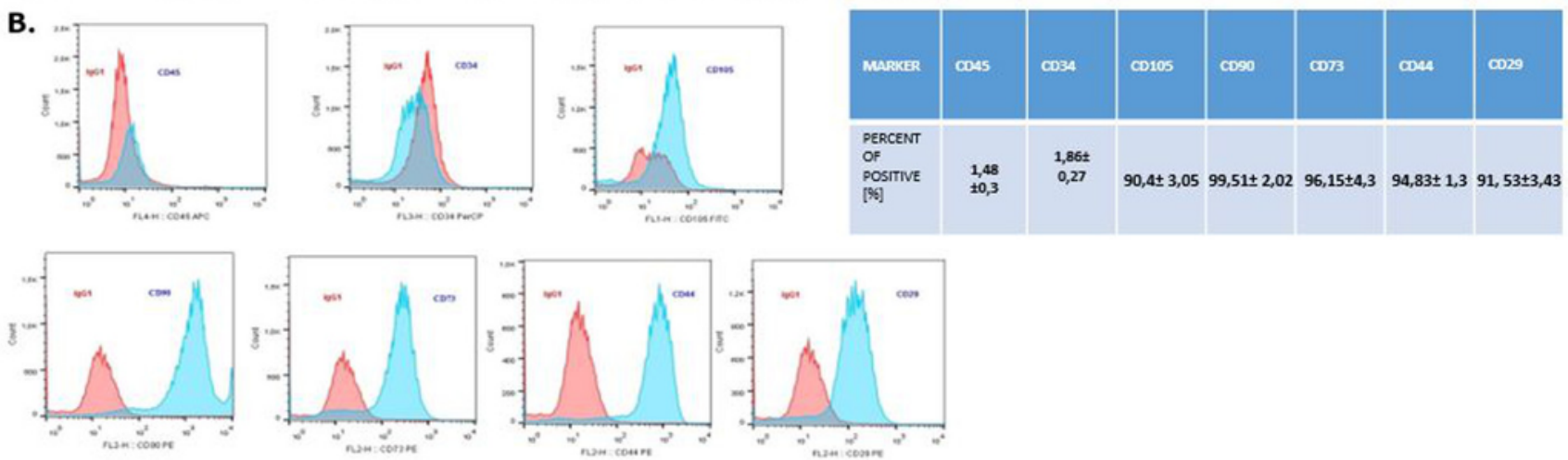

C.

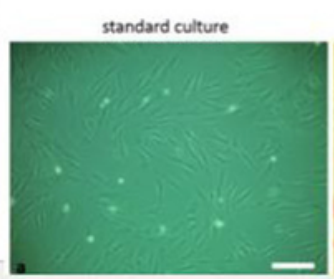

osteogenic stimulation

adipogenic stimulation

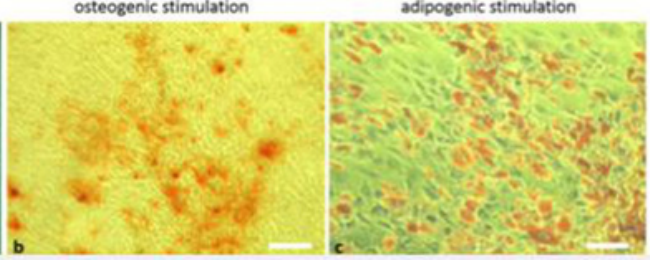


3

proliferation factor and PDT chondrogenesis

Chondrogenesis proliferation factor and population doubling time

A

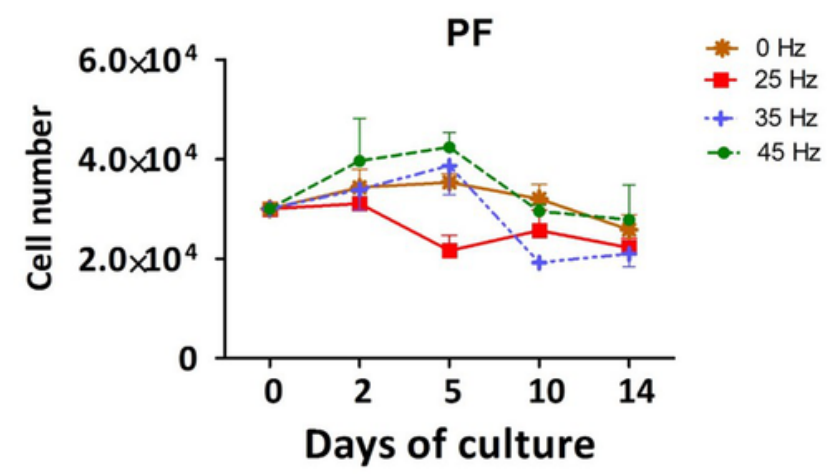

B

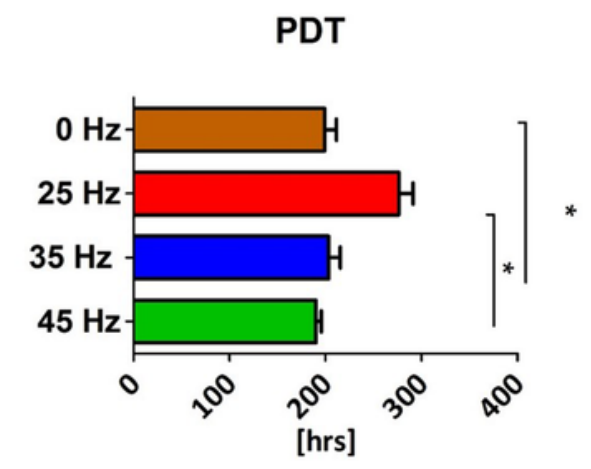


4

qPCR and morphology chondroblasts
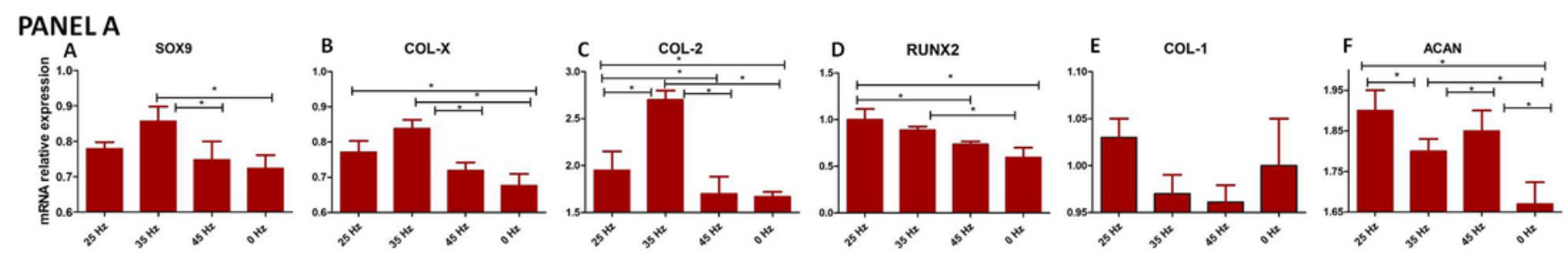

PANEL B

$25 \mathrm{HZ}$

$35 \mathrm{~Hz}$

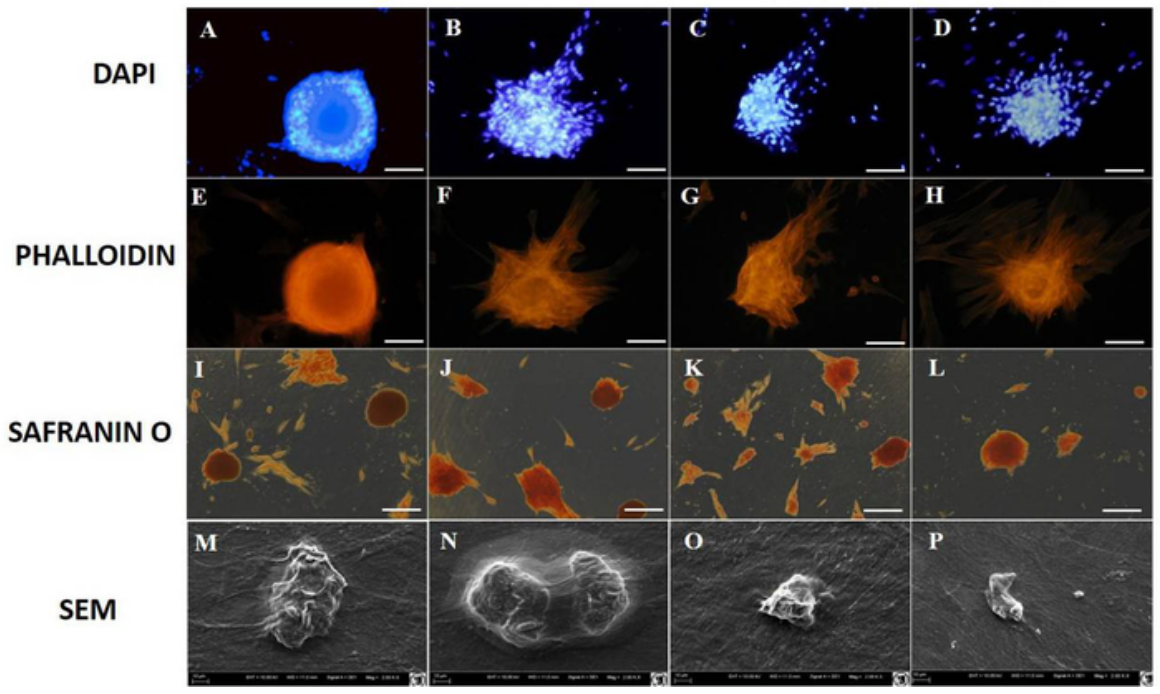

$45 \mathrm{~Hz}$

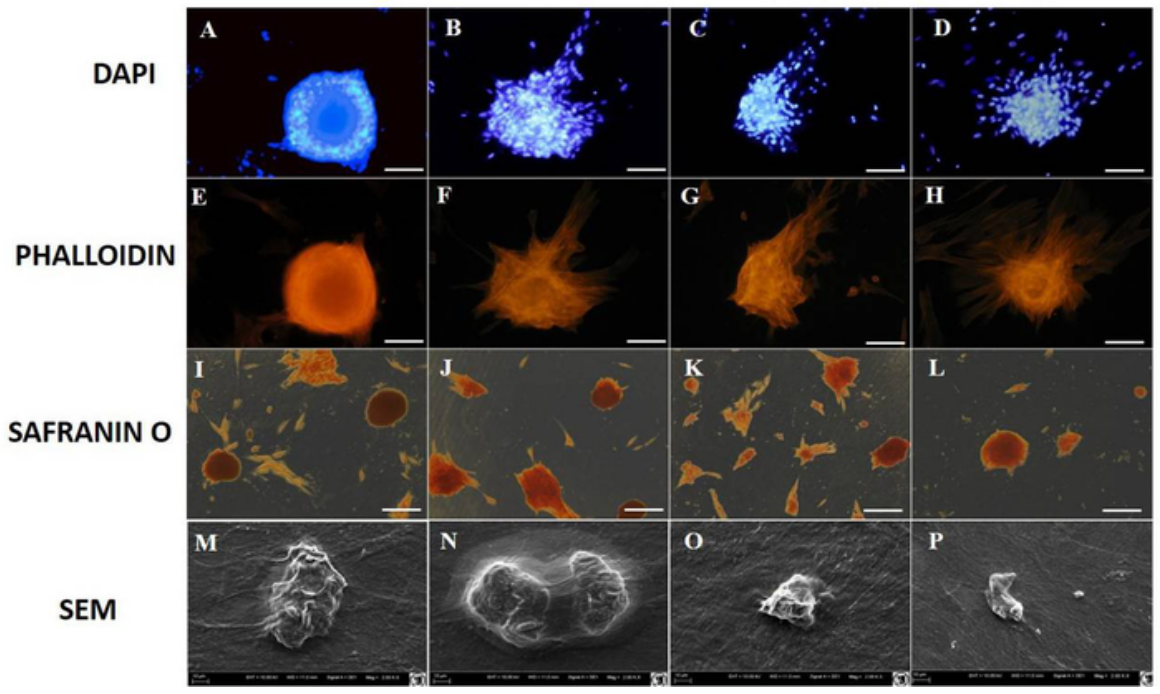

$\mathrm{OHz}$

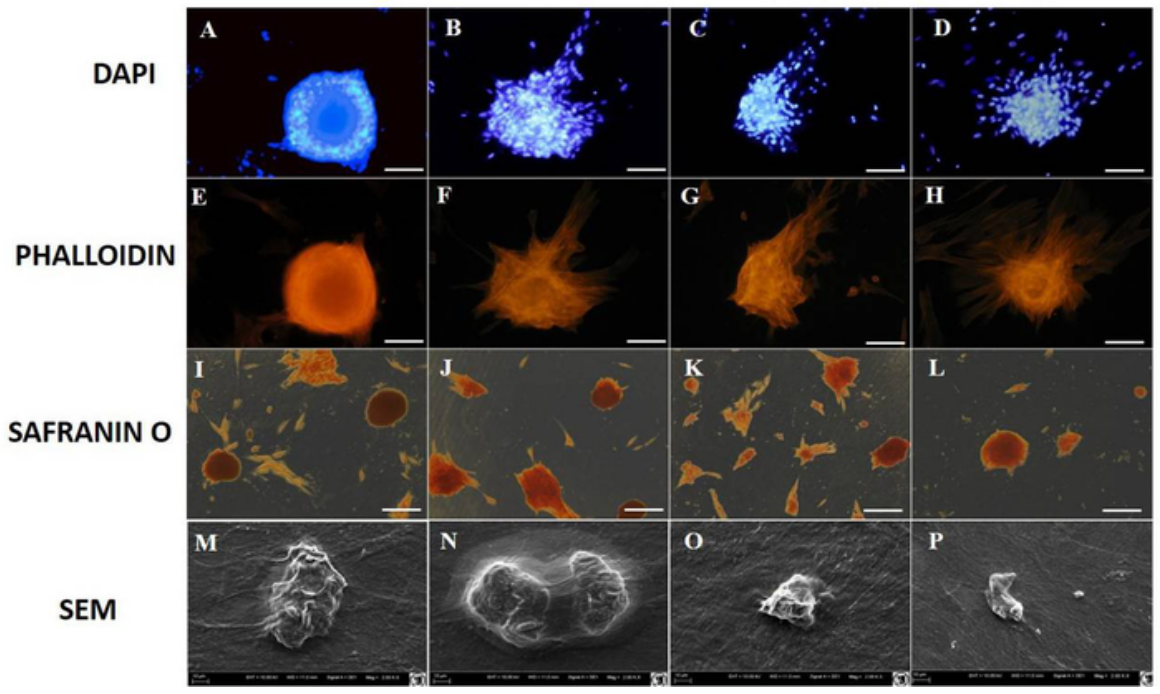




\section{5}

ELISA and chondro nodules

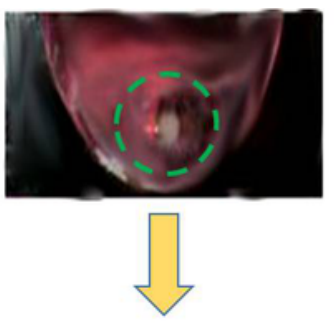

PANEL A
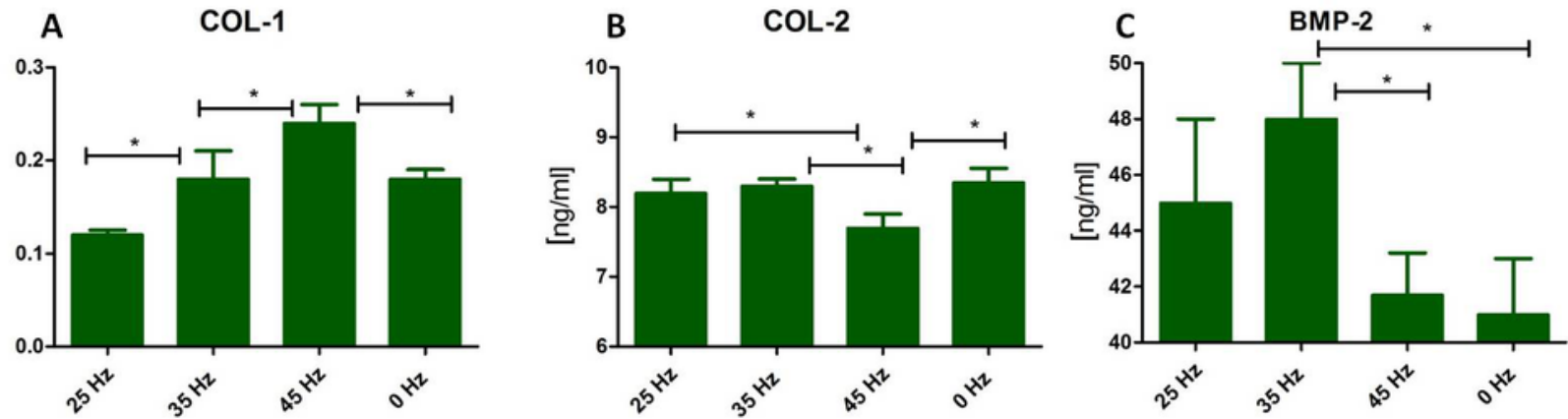

PANEL B

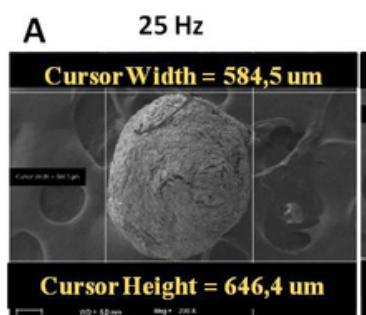

B $\quad 35 \mathrm{~Hz}$

C

$45 \mathrm{~Hz}$

D $\quad 0 \mathrm{~Hz}$

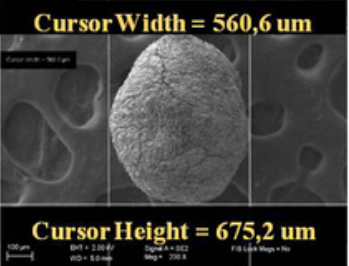

Cursor Width $=714,3$ um

Cursor Width $=\mathbf{5 4 7 , 1}$ um

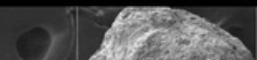

(f) 
6

safranin absorbtion and diametres of chondronodules

A

Percentage of Safranin staining absorption

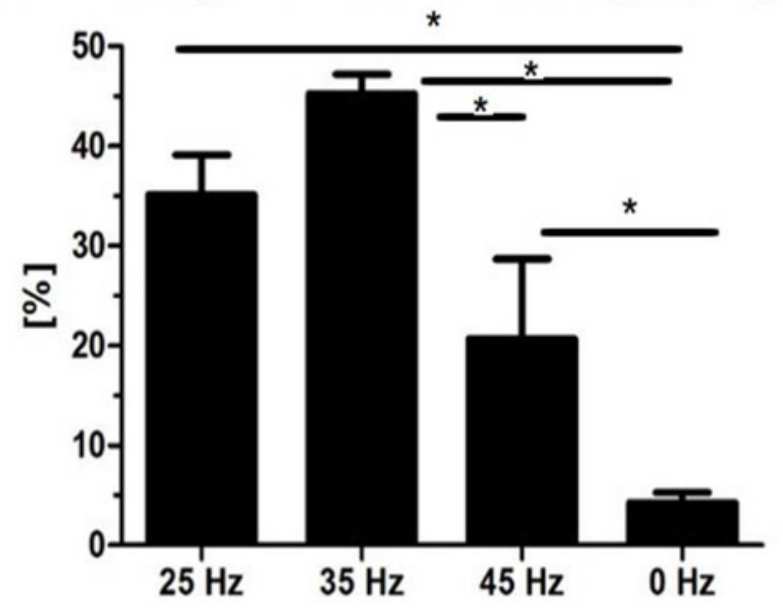

B

The average diameter of nodules

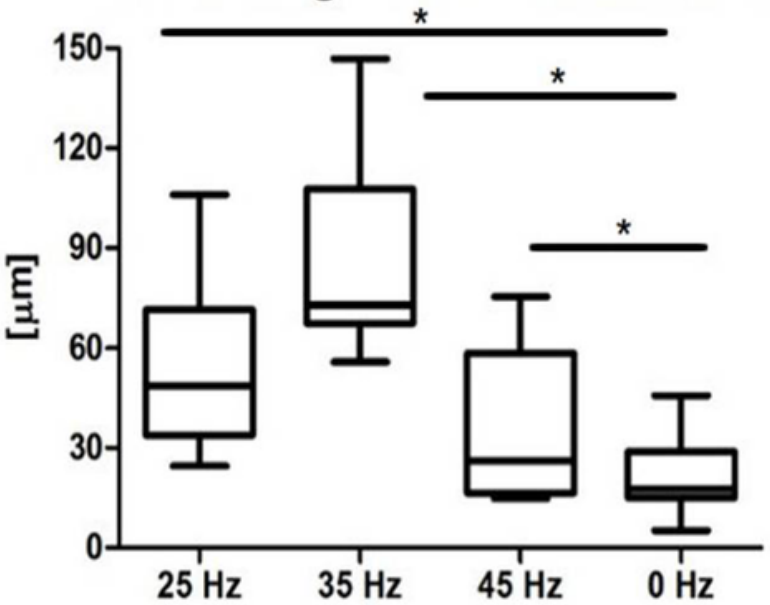


7

Integrin expression (qPCR)

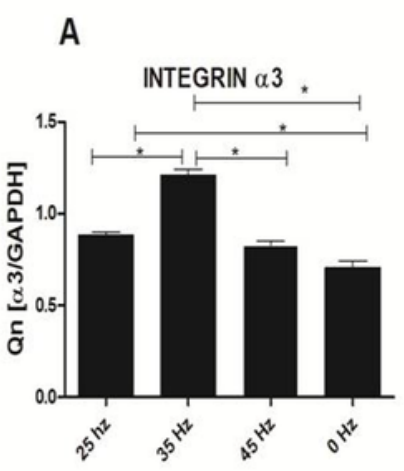

B

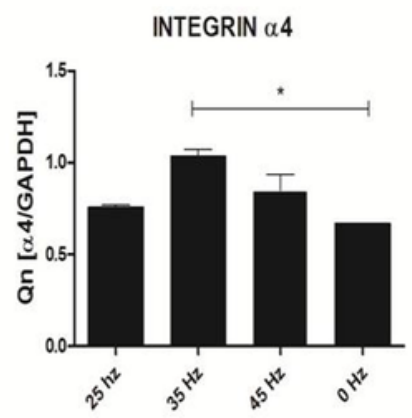

C

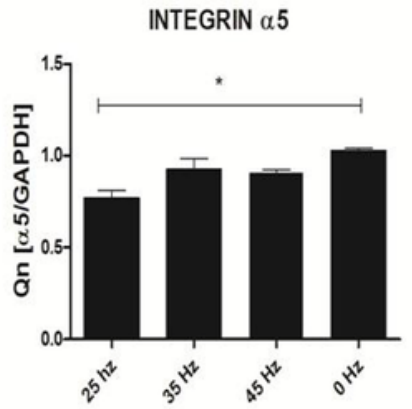

D

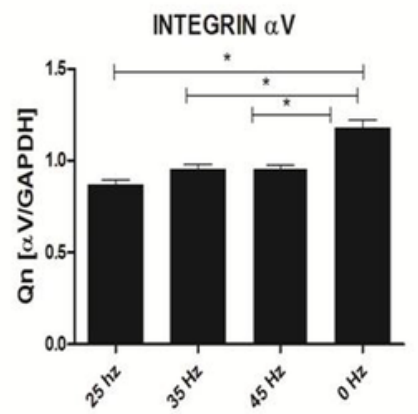

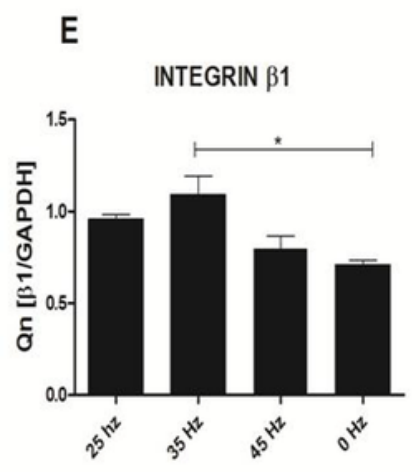
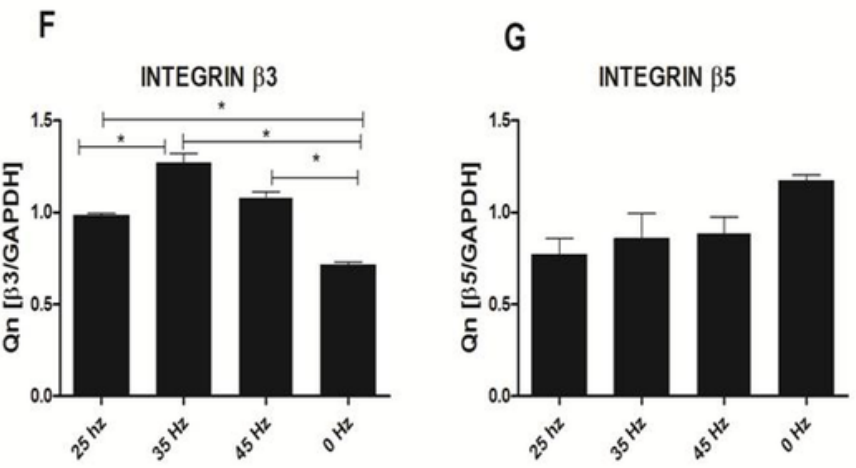
8

proliferation factor and PDT in adipogenesis

\section{Adipogenesis proliferation factor and population doubling time}

B

A

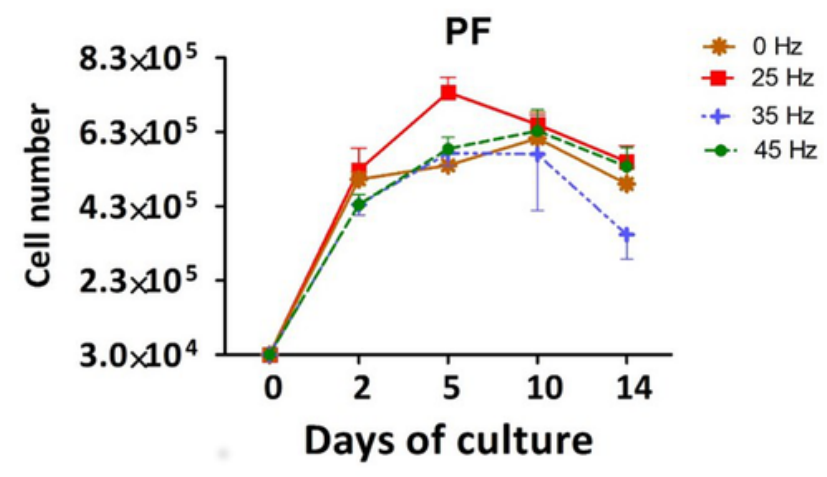

PDT

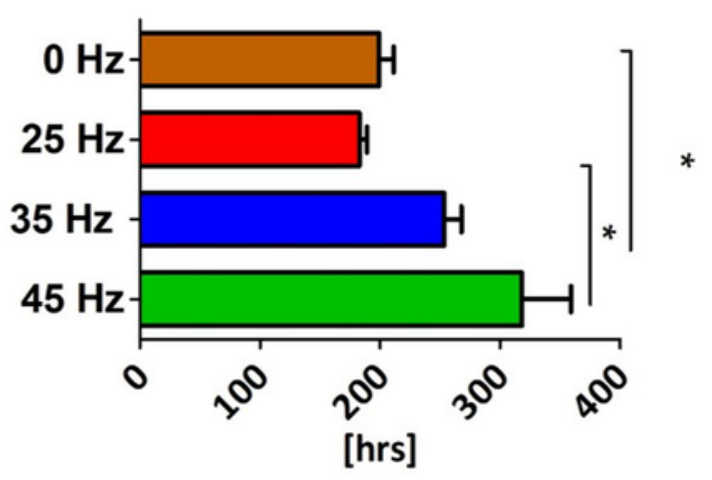


9

qPCR and morphology oof adipocytes

PANEL A
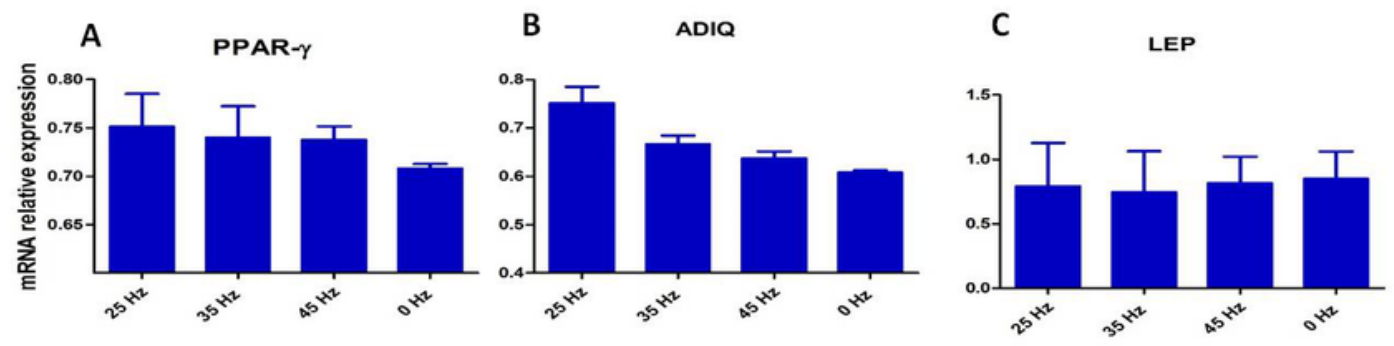

PANEL B

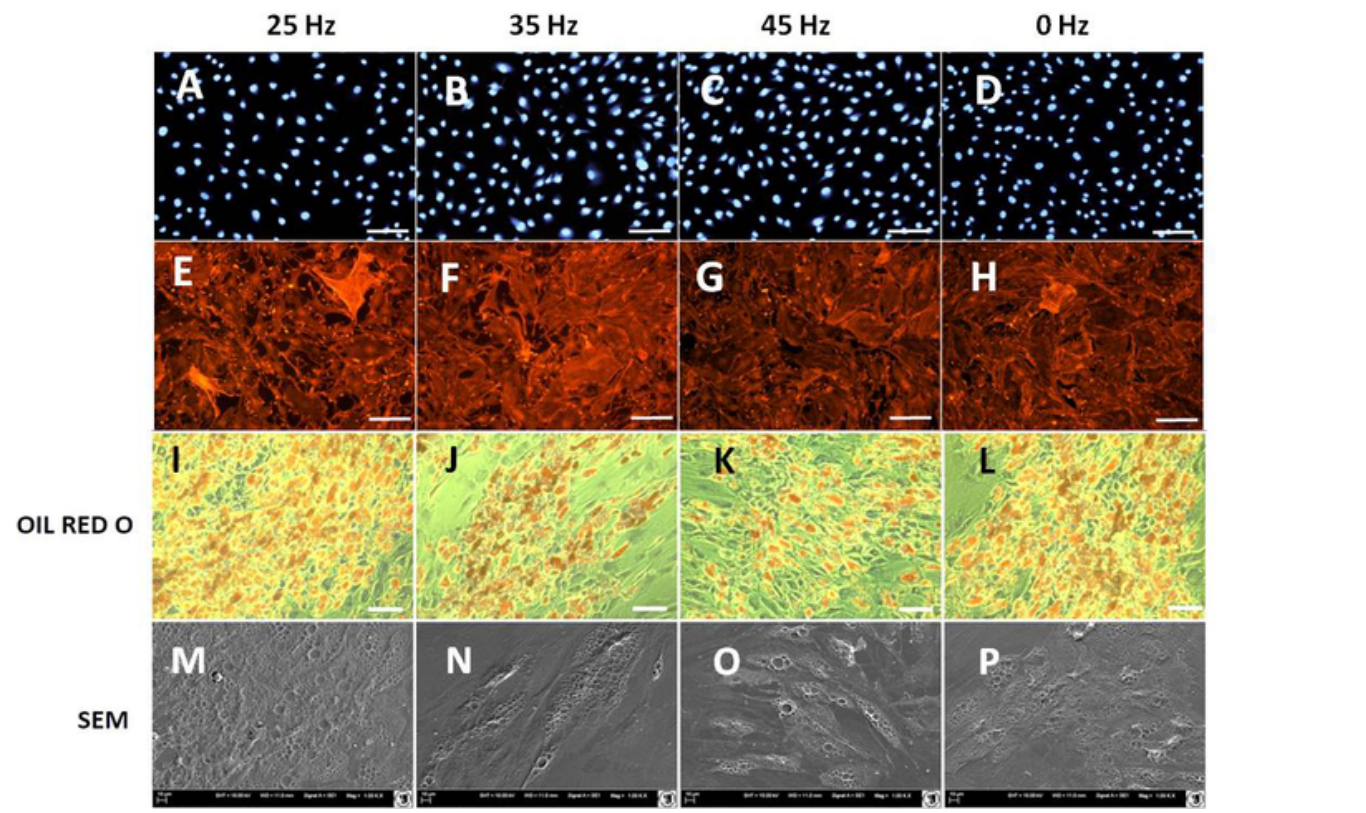


10

Oil red $\mathrm{o}$ absorption and adipocytes diameters

A Percentage of Oil Red 0 staining absorption

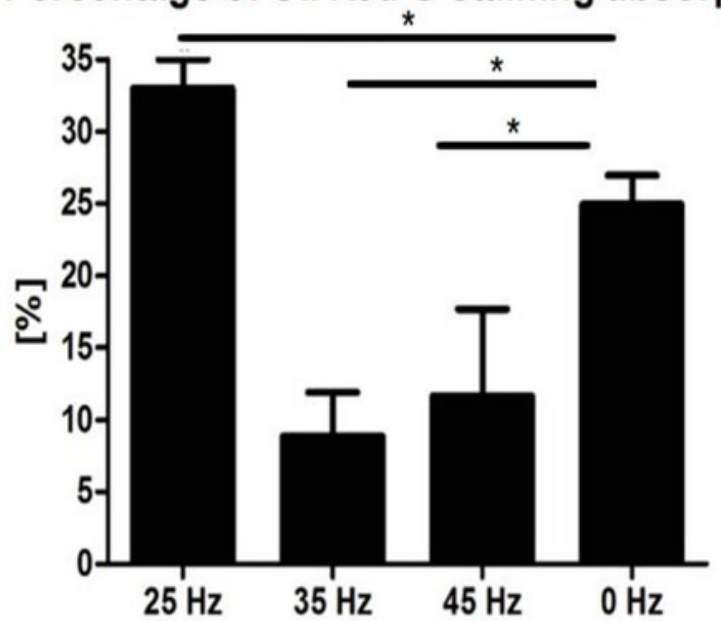

B

The average diameter of adipocytes

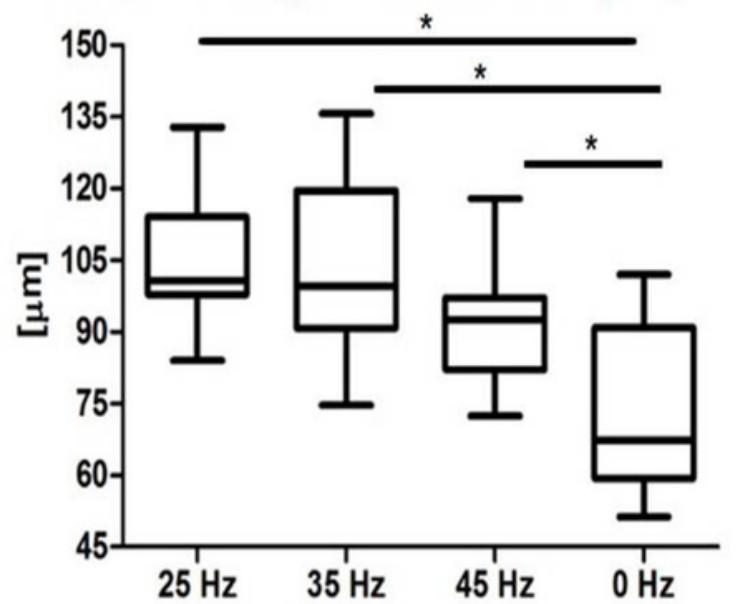

